\title{
A CONTROL PROBLEM FOR BURGERS' EQUATION WITH BOUNDED INPUT/OUTPUT
}

\author{
John A. Burns ${ }^{1}$ \\ Interdisciplinary Center for Applied Mathematics \\ Department of Mathematics \\ Virginia Polytechnic Institute and State University \\ Blacksburg, VA 24061 \\ and \\ Center for Applied Mathematical Sciences \\ Department of Mathematics \\ University of Southern California \\ Los Angeles, CA 90089 \\ Sungkwon Kang ${ }^{2}$ \\ Interdisciplinary Center for Applied Mathematics \\ Department of Mathematics \\ Virginia Polytechnic Institute and State University \\ Blacksburg, VA 24061
}

\begin{abstract}
A stabilization problem for Burgers' equation is considered. Using linearization, various controllers are constructed which minimize certain weighted energy functionals. These controllers produce the desired degree of stability for the closed-loop nonlinear system. A numerical scheme for computing the feedback gain functional is developed and several numerical experiments are performed to show the theoretical results.
\end{abstract}

\footnotetext{
${ }^{1}$ Research supported by the National Aeronautics and Space Administration under NASA Contract No. NAS1-18605 while the author was in residence at the Institute for Computer Applications in Science and Engineering (ICASE), NASA Langley Research Center, Hampton, VA 23665. Research also supported in part by the Air Force Office of Sientific Research under grant AFOSR 89-0001 and the Defense Advanced Research Projects Agency under contracts F49620-87-C-0116 and N00014-88-K-0721.

${ }^{2}$ This research was supported in part by the Defense Advanced Research Projects Agency under contract F49620-87-C-0116.
} 



\section{Introduction}

Burgers' equation is a one-dimensional simple model for convection-diffusion phenomena such as shock waves, supersonic flow about airfoils, traffic flows, acoustic transmission, etc. For high Reynolds number, the solution of Burgers' equation produces steep gradients due to the nonlinear nature of the convection.

In this paper, we consider a stabilization problem for Burgers' equation. We employ " $\alpha$-shifted" linear feedback control laws to obtain a desired degree of stability, on a certain energy space, for the closed-loop nonlinear system.

Burgers' equation

$$
\frac{\partial}{\partial t} z(t, x)+z(t, x) \frac{\partial}{\partial x} z(t, x)=\epsilon \frac{\partial^{2}}{\partial x^{2}} z(t, x)
$$

was introduced by Burgers [5,6,7] as a simple model for turbulence, where $\epsilon>0$ is a viscosity coefficient. Since then, many researchers have considered the conservation law

$$
\frac{\partial}{\partial t} z(t, x)+z(t, x) \frac{\partial}{\partial x} z(t, x)=0
$$

and the "viscosity solution"

$$
z(t, x)=\lim _{\epsilon \downarrow 0} z^{\epsilon}(t, x)
$$

where $z^{\epsilon}(t, x)$ satisfies equation (1.1), see $[9,16,18,22,24,25,26]$.

Oleinik [26] proved that for any $L^{\infty}$-initial data, there is a unique viscosity solution for equation (1.2) and the solution satisfies the "entropy condition"

$$
\frac{z(t, x+a)-z(t, x)}{a}<\frac{E}{t}
$$

for all $t>0, a>0,-\infty<x<\infty$ and for some constant $E>0$. A complete discussion of these results may be found in [32].

Almost no results exist for the control problem associated with Burgers' equation. Chen, Wang and Weerakoon $[8,35]$ considered an optimal control problem for equation (1.2) with $-\infty<x<\infty$. The problem was to select an initial function to minimize a specific cost functional $J$. They obtained sufficient conditions for the differentiability of $J$ with respect to 
the initial function and explicit expression of the entropy solution of (1.2) in terms of initial data.

In this paper we consider a control problem for Burgers' equation (1.1) defined on a finite interval. Specifically, we will find several feedback laws stabilizing the nonlinear system (1.1) with a certain exponential decay rate. The feedback laws will be obtained from the linearized equation. Curtain [11] has considered a stabilization problem for certain semilinear evolution equations. Using Kielhöfer's stability results for semilinear evolution equations [20], she showed that, under certain conditions, there exists a finite dimensional compensator which produces a stable closed-loop system. These finite dimensional compensators are also obtained from the linearized control system. Applying her results to Burgers' equation (1.1) with, for example, Dirichlet boundary conditions, one can obtain the stabilizability results of the closed-loop system which are similar to ours. However, in [11], there is a restriction on the action of the output operators. The domain of the output operator was required to be a certain subspace of $L^{2}$ which contains the Sobolev space $H_{0}^{1}$. In this paper, we investigate optimal feedback laws, instead of finite dimensional compensators, in the sense that they minimize certain energy functionals.

Well-posedness and stability results for the open-loop system are obtained in Section 2 . In Section 3, a "shifted" linear control problem, (LQR) $\hat{\alpha}$, is introduced. Under appropriate selection of input and output operators, $(\mathrm{LQR})_{\alpha} \hat{\alpha}$ is stabilizable and detectable. The feedback control law obtained from (LQR) $\alpha$ produces the desired degree of stability for the closedloop nonlinear system, (Theorem 3.10). In Section 4, a numerical scheme for computing the "feedback functional gains" is developed and several numerical experiments are performed.

We shall use standard notation. If $\left(X,\|\cdot\|_{X}\right)$ and $\left(Y,\|\cdot\|_{Y}\right)$ are normed linear spaces, then $\mathcal{C}(X, Y)$ will denote the space of all bounded linear operators from $X$ to $Y$ and for any $A \in \mathcal{L}(X, Y),\|A\|$ or $\|A\|_{\mathcal{L}(X, Y)}$ will denote the operator norm on the space $\mathcal{L}(X, Y)$. In the event that $X=Y$ we denote $\mathcal{L}(X, Y)$ by $\mathcal{L}(X)$. From time to time we will use $\|\cdot\|$ without any subindex for vector or operator norm. In all such cases the appropriate index for $\|\cdot\|$ will be understood from the context. For a Hilbert space $X$, we denote the inner product on $X \times X$ by $\left\langle\cdot, \cdot>_{X}\right.$. Given a linear operator $A$ from $X$ into itself, we denote its domain, spectrum, resolvent and adjoint by $\mathcal{D}(A), \sigma(A), \rho(A)$ and $A^{*}$, respectively. For real numbers $a, b$ with $a<b, L^{p}(a, b ; X), 1<p<\infty$, will be the space of all Lebesgue 
measurable functions $f$ from $(\mathrm{a}, \mathrm{b})$ to $X$ such that $\|f\|_{L^{p}(a, b)}=\left(\int_{a}^{b}|f(x)|^{p} d x\right)^{\frac{1}{p}}<\infty$. The spaces $H^{k}(a, b)$ and $H_{0}^{k}(a, b)$ are the standard Sobolev spaces defined by $H^{k}(a, b)=\{f \in$ $\left.L^{2}(a, b) \mid f^{(j)} \in L^{2}(a, b), j=0,1, \cdots, k\right\}$ and $H_{0}^{k}(a, b)=\left\{f \in H^{k}(a, b) \mid f^{(j)}(a)=f^{(j)}(b)=\right.$ $0, j=0,1, \cdots, k-1\}$, respectively. The dual space $H^{-k}(a, b)$ of $H_{0}^{k}(a, b)$ is the space of all continuous linear functionals on $H_{0}^{k}(a, b)$ represented by the inner product $\langle\cdot, \cdot\rangle_{L^{2}(a, b)}$.

Finally, we present a physical example that may be found in most standard references to motivate the control problem for Burgers' equation. Other examples involving traffic flows, supersonic flow about airfoils, acoustic transmission and turbulence in hydrodynamic flows can be found in [12] and the references given there. The following example is taken almost directly from [12].

\section{Example (Shock Waves)}

An impulsively-started piston moving at a constant velocity into a tube containing a compressible fluid initially at rest creates compression waves. The compression waves eventually coalesce, due to the nonlinear nature of the convection, to form a single shock wave. The one-dimensional unsteady motion of the fluid is governed by the continuity equation

$$
\frac{\partial}{\partial t} \rho(t, x)+\rho(t, x) \frac{\partial}{\partial x} v(t, x)+v(t, x) \frac{\partial}{\partial x} \rho(t, x)=0
$$

and the $x$-momentum equation

$$
\frac{\partial}{\partial t} v(t, x)+v(t, x) \frac{\partial}{\partial x} v(t, x)+\left(\frac{\partial}{\partial x} p(t, x)\right) / \rho(t, x)=\delta \frac{\partial^{2}}{\partial x^{2}} v(t, x)
$$

where $\rho$ is the density, $v$ is the velocity, $p$ is the pressure and $\delta$ is the "diffusivity of sound". It is convenient to replace the density by the sound speed, $a=a(t, x)$ by $a(t, x) / a_{0}=$ $\left(\rho(t, x) / \rho_{0}\right)^{\frac{\gamma-1}{2}}$, where $\gamma>1$ is the specific heats ratio and the subscript 0 refers to the undisturbed values [23]. Equations (1.5) and (1.6) become

$$
\frac{\partial}{\partial t} a(t, x)+v(t, x) \frac{\partial}{\partial x} a(t, x)+\frac{\gamma-1}{2} a(t, x) \frac{\partial}{\partial x} v(t, x)=0
$$

and

$$
\frac{\partial}{\partial t} v(t, x)+v(t, x) \frac{\partial}{\partial x} v(t, x)+\frac{2}{\gamma-1} a(t, x) \frac{\partial}{\partial x} a(t, x)=\delta \frac{\partial^{2}}{\partial x^{2}} v(t, x)
$$


where $\delta$ is a function of the undisturbed (to the right of the shock) viscosity, density, specific heat and thermal conductivity of the medium. Equations (1.7) and (1.8) can be simplified by introducing the Riemann invariants,

$$
r(t, x)=\frac{a(t, x)}{\gamma-1}+\frac{v(t, x)}{2}, \quad s(t, x)=\frac{a(t, x)}{\gamma-1}-\frac{v(t, x)}{2}
$$

to give

$$
\frac{\partial}{\partial t} r(t, x)+(a(t, x)+v(t, x)) \frac{\partial}{\partial x} r(t, x)=\frac{\delta}{2} \frac{\partial^{2}}{\partial x^{2}}(r(t, x)-s(t, x))
$$

and

$$
\frac{\partial}{\partial t} s(t, x)-(a(t, x)-v(t, x)) \frac{\partial}{\partial x} s(t, x)=\frac{\delta}{2} \frac{\partial^{2}}{\partial x^{2}}(s(t, x)-r(t, x)) .
$$

Consider the propagation of a disturbance into an initially undisturbed region, $s=s_{0}$ where $s_{0}=\frac{a_{0}}{\gamma-1}$. Then the problem is governed by equation (1.10). But from equation (1.9),

$$
a(t, x)+v(t, x)=\frac{\gamma+1}{2} r(t, x)+\frac{\gamma-3}{2} s_{0},
$$

thus equation (1.10) becomes

$$
\frac{\partial}{\partial t} r(t, x)+\left(\frac{\gamma+1}{2} r(t, x)+\frac{\gamma-3}{2} s_{0}\right) \frac{\partial}{\partial x} r(t, x)=\frac{\delta}{2} \frac{\partial^{2}}{\partial x^{2}} r(t, x) .
$$

As the final step we introduce the change of variables

$$
z(t, x)=\frac{\gamma+1}{2}\left(r(t, x)-r_{0}\right), \quad \xi=x-a_{0} t
$$

to give Burgers' equation

$$
\frac{\partial}{\partial t} z(t, \xi)+z(t, \xi) \frac{\partial}{\partial \xi} z(t, \xi)=\frac{\delta}{2} \frac{\partial^{2}}{\partial \xi^{2}} z(t, \xi)
$$

From equations (1.12) and (1.14) we have $z(t, \xi)=\{a(t, \xi)+v(t, \xi)\}-\left\{v_{0}+a_{0}\right\}$, where $v_{0}=0$, i.e., $u(t, \xi)$ is the excess wavelet velocity (the difference between propagation speeds of disturbance in stagnation and nonstagnation conditions). The coordinate $\xi$ is measured relative to a frame of reference moving with the undisturbed speed of sound $a_{0}$. 


\section{Well-posedness and stability of Burgers' equation}

In this section, we consider well-posedness and stability properties of the solution for Burgers' equation with Dirichlet boundary condition. These results will be needed in the analyses of our control problems in the next section. We first consider an abstract version of this problem and then specialize to Burgers' equation.

Consider an initial value problem

$$
\frac{d}{d t} z(t)=\mathcal{A} z(t)+f(t, z(t)), \quad z\left(t_{0}\right)=z_{0}, \quad\left(t>t_{0}\right),
$$

on a Hilbert space $X$, where $\mathcal{A}$ is the infinitesimal generator of an analytic semigroup $S(t)$ satisfying $\|S(t)\|_{\mathcal{L}(X)} \leq M e^{\omega t}, t \geq t_{0}$, for some constants $M=M(\omega) \geq 1$ and $\omega \geq 0$. Since $S(t)$ is analytic, the fractional powers of $\mathcal{A}_{1}=-\mathcal{A}+a I$ are well-defined for any $a>\omega[27$, Chapters 1,2]. Since $0 \in \rho\left(\mathcal{A}_{1}\right)$, the resolvent of $\mathcal{A}_{1}, \mathcal{A}_{1}^{\mu}$ is invertible for all $0 \leq \mu \leq 1$. Therefore, the graph norm $\|z\|+\left\|\mathcal{A}_{1}^{\mu} z\right\|$ on the domain $\mathcal{D}\left(\mathcal{A}_{1}^{\mu}\right)$ of $\mathcal{A}_{1}^{\mu}$ is equivalent to the norm $\|z\|_{\mu}=\left\|\mathcal{A}_{1}^{\mu} z\right\|$. We denote the Hilbert space $\mathcal{D}\left(\mathcal{A}_{1}^{\mu}\right)$ with the norm $\|z\|+\left\|\mathcal{A}_{1}^{\mu} z\right\|$ or $\left\|\mathcal{A}_{1}^{\mu} z\right\|$ by $\left(X_{\mu},\|\cdot\|_{\mu}\right)$.

We shall make the following assumption $[17,27]$.

Hypothesis (F): Let $U$ be an open subset of $\left[t_{0}, \infty\right) \times X_{\mu}$. The function $f: U \longrightarrow X$ satisfies the hypothesis $(\mathrm{F})$ if for every $(t, z) \in U$ there is a neighborhood $V \subset U$ and constants $L \geq 0,0<\theta \leq 1$ such that

$$
\left\|f\left(t_{1}, z_{1}\right)-f\left(t_{2}, z_{2}\right)\right\|_{X} \leq L\left(\left|t_{1}-t_{2}\right|^{\theta}+\left\|z_{1}-z_{2}\right\|_{\mu}\right)
$$

for all $\left(t_{i}, z_{i}\right) \in V, i=1,2$, i.e., $f$ is locally Hölder continuous in $t$, locally Lipschitzian in $z$, on $U$.

Now we are ready to state the local existence theorem for the solution of equation (2.1). The following result appears as Theorem 3.3.3 in [17].

Theorem 2.1. Let $\mathcal{A}$ be as before and $f$ satisfy hypothesis $(\mathrm{F})$. Then for any $\left(t_{0}, z_{0}\right) \in$ $U \subset \mathbf{R}^{+} \times X_{\mu}$, there exists $T=T\left(t_{0}, z_{0}\right)>0$ such that equation (2.1) has a unique (strong) solution $z(t)$ on $\left[t_{0}, t_{0}+T\right)$ with initial value $z\left(t_{0}\right)=z_{0}$.

Now, consider Burgers' equation, with Dirichlet boundary condition, on a finite interval 
$[0, \ell]$ given by

$$
\begin{aligned}
\frac{\partial}{\partial t} z(t, x) & =\epsilon \frac{\partial^{2}}{\partial x^{2}} z(t, x)-z(t, x) \frac{\partial}{\partial x} z(t, x), \quad 0<x<\ell, \quad t>0 \\
z(t, 0) & =z(t, \ell)=0 \\
z(0, x) & =z_{0}(x)
\end{aligned}
$$

where $\epsilon=\frac{1}{\operatorname{Re}}>0$ and Re is the Reynolds number. In order to place the system (2.3) into a semigroup framework let $z(t)(\cdot)=z(t, \cdot), z_{0}(\cdot)=z(0, \cdot)$ and $H=L^{2}(0, \ell)$. Define the operator $A_{\epsilon}$ by

$$
A_{\epsilon} \phi=\epsilon \phi^{\prime \prime}
$$

for all $\phi \in \mathcal{D}\left(A_{\epsilon}\right)=H^{2}(0, \ell) \cap H_{0}^{1}(0, \ell)$. The system $(2.3)$ can now be written as the initial value problem

$$
\frac{d}{d t} z(t)=A_{c} z(t)+f(t, z(t)), \quad z(0)=z_{0}, \quad(t>0)
$$

on the space $H$, where $f(t, z)=-z z^{\prime}$ is defined on the space $H_{0}^{1}(0, \ell)$. It is well-known $[17,27,34]$ that $A_{\epsilon}$ generates an analytic semigroup $S(t)$ on $H$.

We summarize the basic properties of the infinitesimal generator $A_{\epsilon}$ and its semigroup $S(t), t \geq 0$, in the following remark.

Remark 2.2. (i) The spectrum $\sigma\left(A_{\epsilon}\right)$ of $A_{\epsilon}$ consists of all eigenvalues $\lambda_{n}=-\epsilon n^{2} \pi^{2} / \ell^{2}$, $n=1,2, \cdots$, and for each eigenvalue $\lambda_{n}$ the corresponding eigenfunction $\phi_{n}$ is given by

$$
\phi_{n}(x)=\sqrt{2} \sin \frac{n \pi}{\ell} x, \quad 0<x<\ell .
$$

(ii) The operator $A_{\epsilon}$ is self-adjoint, i.e., $A_{\epsilon}=A_{\epsilon}^{*}$, and the semigroup $S(t)$ can be represented by the following formula

$$
S(t) z=\sum_{n=1}^{\infty} e^{-\left(\epsilon n^{2} \pi^{2} / \ell^{2}\right) t}<z, \phi_{n}>\phi_{n}
$$

for all $z \in H$, where $\phi_{n}$ 's are defined by equation (2.6). Moreover, from equation (2.7), it is easy to see that $S(t)$ has the stability property

$$
\|S(t)\|_{\mathcal{C}(H)} \leq e^{-\left(\epsilon \pi^{2} / \ell^{2}\right) t}, \quad t \geq 0 .
$$


A simple application of Schwartz inequality gives the following first Poincaré inequality [36, p. 116].

Lemma 2.3. For any $z \in H_{0}^{1}(0, \ell)$,

$$
\|z\|_{H} \leq \ell\left\|z^{\prime}\right\|_{H}
$$

where $H=L^{2}(0, \ell)$.

Remark 2.4. (i) The above lemma gives an equivalent norm $\|z\|_{H_{0}^{1}} \equiv\left\|z^{\prime}\right\|_{L^{2}}$ on the space $H_{0}^{1}(0, \ell)$.

(ii) It is well-known that $\mathcal{D}\left(\left(-A_{\epsilon}\right)^{\frac{1}{2}}\right)=H_{0}^{1}(0, \ell)[17$, p. 29], $[21$, p. 326].

Lemma 2.5. For any $z \in H_{0}^{1}(0, \ell)=\mathcal{D}\left(\left(-A_{\epsilon}\right)^{\frac{1}{2}}\right)$, the following inequalities hold.

$$
\begin{aligned}
& \|S(t) z\|_{H_{0}^{1}} \leq e^{-\gamma t}\|z\|_{H_{0}^{1}}, \quad(t \geq 0) \\
& \|S(t) z\|_{H_{0}^{1}} \leq\left(\frac{1+\ell}{\sqrt{2 \epsilon}} \frac{1}{\sqrt{t}}+\frac{\pi(1+\ell)}{\ell}\right) e^{-\gamma t}\|z\|_{L^{2}(0, \ell)}, \quad(t>0),
\end{aligned}
$$

where $\gamma=\epsilon \pi^{2} / \ell^{2}$.

Proof: For any $z \in H_{0}^{1}(0, \ell)$, we know that $\left(-A_{\epsilon}\right)^{\frac{1}{2}} S(t) z=S(t)\left(-A_{\epsilon}\right)^{\frac{1}{2}} z$. Hence, by Remark 2.2,

$$
\|S(t) z\|_{H_{0}^{1}}=\|S(t) z\|_{L^{2}}+\left\|\left(-A_{\epsilon}\right)^{\frac{1}{2}} S(t) z\right\|_{L^{2}} \leq\|S(t)\|\left(\|z\|+\left\|\left(-A_{\epsilon}\right)^{\frac{1}{2}} z\right\|\right) \leq e^{-\gamma t}\|z\|_{H_{0}^{1}}
$$

The inequality (2.11) follows from Remark 2.2 , Lemma 2.3 and the estimate

$$
\begin{aligned}
\|S(t) z\|_{H_{0}^{1}} & \leq(1+\ell)\left\|\left(-A_{\epsilon}\right)^{\frac{1}{2}} S(t) z\right\|_{H}=(1+\ell)\left\|\left(-A_{\epsilon}\right)^{\frac{1}{2}} \sum_{n=1}^{\infty} e^{\lambda_{n} t}<z, \phi_{n}>\phi_{n}\right\| \\
& =(1+\ell)\left\|\sum_{n=1}^{\infty} \frac{n \pi}{\ell} e^{\lambda_{n} t}<z, \phi_{n}>\sqrt{2} \cos \frac{n \pi}{\ell} x\right\| \\
& \leq(1+\ell)\left(\sup \left\{\frac{n \pi}{\ell} e^{\left(\lambda_{n}+\gamma\right) t}: n=1,2, \ldots\right\}\right) e^{-\gamma t}\|z\|_{H}
\end{aligned}
$$

and

$$
\sup \left\{\frac{n \pi}{\ell} e^{\left(\lambda_{n}+\gamma\right) t}: n=1,2, \cdots\right\} \leq \begin{cases}\frac{1}{\sqrt{2 \epsilon}} \frac{1}{\sqrt{t}}, & 0<t \leq \frac{\ell^{2}}{2 \epsilon \pi^{2}} \\ \frac{\pi}{\ell}, & t \geq \frac{\ell^{2}}{2 \epsilon \pi^{2}}\end{cases}
$$

where $\lambda_{n}=-\epsilon n^{2} \pi^{2} / \ell^{2}$ and $\phi_{n}(x)=\sqrt{2} \sin \frac{n \pi}{\ell} x, n=1,2, \cdots$ 
Remark 2.6. The inequality (2.11) holds for every $z \in H=L^{2}(0, \ell)$, since the semigroup $S(t)$ is analytic.

Now we have the well-posedness and stability properties of Burgers' equation (2.3) on the space $H_{0}^{1}(0, \ell)$. The following theorem follows from an application of Theorem 5.1.1 in [17].

Theorem 2.7. For any given $\beta>0,0<\beta<\gamma=\epsilon \pi^{2} / \ell^{2}$, there is a $\rho=\rho(\ell, \epsilon, \beta)>0$ such that for any initial data $z_{0} \in H_{0}^{1}(0, \ell)$, with $\left\|z_{0}\right\|_{H_{0}^{1}} \leq \frac{\rho}{2}$, there is a unique solution $z(t)=z\left(t, 0 ; z_{0}\right) \in H_{0}^{1}(0, \ell)$ of equation (2.3). Moreover, the solution satisfies the inequality

$$
\left\|z\left(t, 0 ; z_{0}\right)\right\|_{H_{0}^{1}} \leq 2 e^{-\beta t}\left\|z_{0}\right\|_{H_{0}^{1}} \quad(t \geq 0)
$$

and $\rho=\rho(\ell, \epsilon, \beta)>0$ can be chosen to satisfy

$$
0<\rho<\frac{\sqrt{\epsilon} \ell(\gamma-\beta)}{\sqrt{2 \pi}(1+\ell)(\ell \sqrt{\gamma-\beta}+\sqrt{2 \pi \epsilon})} .
$$

Proof: Note that 0 is an equilibrium point for the system (2.5). Since $\mathcal{D}\left(\left(-A_{\epsilon}\right)^{\frac{1}{2}}\right)=H_{0}^{1}$, if the nonlinear term $f(z)=-z z^{\prime}$ satisfies the hypothesis (F) with index $\mu=\frac{1}{2}$, then, by Theorem 2.1, we have a unique local solution $z\left(t, 0 ; z_{0}\right)$ on the space $H_{0}^{1}$. It is easy to see that $\left\|f\left(z_{1}\right)-f\left(z_{2}\right)\right\|_{L^{2}} \leq\left(\left\|z_{1}\right\|_{H_{0}^{1}}+\left\|z_{2}\right\|_{H_{0}^{1}}\right)\left\|z_{1}-z_{2}\right\|_{H_{0}^{1}}$ for all $z_{1}, z_{2} \in H_{0}^{1}$, uniformly in $t>0$. Hence, $f$ satisfies the hypothesis $(\mathrm{F})$.

For the global existence and uniqueness of the solution $z\left(t, 0 ; z_{0}\right) \in H_{0}^{1}$, let $z_{0}$ be any initial data in $H_{0}^{1}$ with $\left\|z_{0}\right\|_{H_{0}^{1}} \leq \frac{\rho}{2}$, where $\rho=\rho(\ell, \epsilon, \beta)$ satisfies the condition (2.13). It follows that

$$
\rho\left\{\frac{1+\ell}{\sqrt{2 \epsilon}} \int_{0}^{\infty} \frac{1}{\sqrt{s}} e^{-(\gamma-\beta) s} d s+\frac{(1+\ell) \pi}{\ell(\gamma-\beta)}\right\}<\frac{1}{2}
$$

since $\int_{0}^{\infty} \frac{1}{\sqrt{s}} e^{-(\gamma-\beta) s} d s=\frac{1}{\sqrt{\gamma-\beta}} \frac{1}{\Gamma\left(\frac{1}{2}\right)} \frac{\pi}{\sin \frac{\pi}{2}}=\frac{\sqrt{\pi}}{\sqrt{\gamma-\beta}}$, where $\Gamma$ is the Gamma function and $\Gamma\left(\frac{1}{2}\right)=$ $\sqrt{\pi}$. Let $\left\|z_{0}\right\|_{H_{0}^{1}} \leq \frac{\rho}{2}$. Then, by the local existence property, there is a unique solution $z\left(t, 0 ; z_{0}\right) \in H_{0}^{1}$ satisfying the inequality $\left\|z\left(t, 0 ; z_{0}\right)\right\|_{H_{0}^{1}}<\rho$ on an interval $\left[0, t_{1}\right)$ for some $t_{1}>0$, where $t_{1}$ is chosen as large as possible. We will show that $t_{1}$ must be infinity. Suppose that $t_{1}$ is finite. Then we must have $\left\|z\left(t_{1}\right)\right\|_{H_{0}^{1}} \geq \rho$. Note that, on the interval $\left[0, t_{1}\right)$,

$$
\|f(z(t))\|_{L^{2}}=\left\|-z(t) z^{\prime}(t)\right\|_{L^{2}} \leq\left(\|z(t)\|_{H_{0}^{1}}\right)^{2} \leq \rho^{2},
$$


where ${ }^{\prime}=\frac{d}{d x}$. Lemma 2.5 , Remark 2.6 together with inequalities $(2.14),(2.15)$ yield

$$
\begin{aligned}
\left\|z\left(t_{1}\right)\right\|_{H_{0}^{1}} & =\left\|S\left(t_{1}\right) z_{0}+\int_{0}^{t_{1}} S\left(t_{1}-s\right) f(z(s)) d s\right\|_{H_{0}^{1}} \\
& \leq\left\|S\left(t_{1}\right) z_{0}\right\|_{H_{0}^{1}}+\int_{0}^{t_{1}}\left\|S\left(t_{1}-s\right) f(z(s))\right\|_{H_{0}^{1}} d s \\
& \leq e^{-\gamma t_{1}}\left\|z_{0}\right\|_{H_{0}^{1}}+\rho^{2} \int_{0}^{t_{1}}\left\{\frac{1+\ell}{\sqrt{2 \epsilon}} \frac{1}{\sqrt{t_{1}-s}}+\frac{(1+\ell) \pi}{\ell}\right\} e^{-\gamma\left(t_{1}-s\right)} d s \\
& \leq \frac{\rho}{2}+\rho^{2}\left\{\frac{1+\ell}{\sqrt{2 \epsilon}} \int_{0}^{\infty} \frac{1}{\sqrt{s}} e^{-\gamma s} d s+\frac{(1+\ell) \pi}{\ell} \int_{0}^{\infty} e^{-\gamma s} d s\right\} \\
& =\frac{\rho}{2}+\rho\left\{\rho\left(\frac{1+\ell}{\sqrt{2 \epsilon}} \sqrt{\frac{\pi}{\gamma}}+\frac{(1+\ell) \pi}{\ell \gamma}\right)\right\}<\frac{\rho}{2}+\frac{\rho}{2}=\rho .
\end{aligned}
$$

This is a contradiction. Therefore, the unique global solution $z\left(t, 0 ; z_{0}\right)$ exists. Moreover, from the above estimate, we know that if $\left\|z_{0}\right\|_{H_{0}^{1}} \leq \frac{\rho}{2}$ then $\left\|z\left(t, 0 ; z_{0}\right)\right\|_{H_{0}^{1}}<\rho$ for all $t \in[0, \infty)$.

Finally, we will derive the stability result $(2.12)$. Let $w(t)=\sup \left\{\|z(s)\|_{H_{0}^{1}} e^{\beta s}: 0 \leq\right.$ $s \leq t\}$. We then have

$$
\begin{aligned}
& \|z(t)\|_{H_{0}^{1}} e^{\beta t} \leq e^{\beta t}\left(\left\|S(t) z_{0}\right\|_{H_{0}^{1}}+\int_{0}^{t}\|S(t-s) f(z(s))\|_{H_{0}^{1}} d s\right) \\
& \leq e^{\beta t}\left(e^{-\gamma t}\left\|z_{0}\right\|_{H_{0}^{1}}+\int_{0}^{t}\left\{\frac{1+\ell}{\sqrt{2 \epsilon}} \frac{1}{\sqrt{t-s}}+\frac{(1+\ell) \pi}{\ell}\right\} e^{-\gamma(t-s)}\|z(s)\|_{H_{0}^{1}}^{2} d s\right) \\
& \leq e^{-(\gamma-\beta) t}\left\|z_{0}\right\|_{H_{0}^{1}}+\rho \int_{0}^{t}\left(\frac{1+\ell}{\sqrt{2 \epsilon}} \frac{1}{\sqrt{t-s}}+\frac{(1+\ell) \pi}{\ell}\right) e^{-(\gamma-\beta)(t-s)}\|z(s)\|_{H_{0}^{1}} e^{\beta s} d s \\
& \leq\left\|z_{0}\right\|_{H_{0}^{1}}+\frac{1+\ell}{\sqrt{2 \epsilon}} \rho\left(\int_{0}^{t} \frac{1}{\sqrt{t-s}} e^{-(\gamma-\beta)(t-s)} d s\right) w(t)+\frac{(1+\ell) \pi}{\ell} \rho\left(\int_{0}^{t} e^{-(\gamma-\beta) s} d s\right) w(t) \\
& \leq\left\|z_{0}\right\|_{H_{0}^{1}}+\rho\left\{\frac{1+\ell}{\sqrt{2 \epsilon}} \int_{0}^{\infty} \frac{1}{\sqrt{s}} e^{-(\gamma-\beta) s} d s+\frac{(1+\ell) \pi}{\ell(\gamma-\beta)}\right\} w(t) \leq\left\|z_{0}\right\|_{H_{0}^{1}}+\frac{1}{2} w(t) .
\end{aligned}
$$

Therefore, $w(t) \leq 2\left\|z_{0}\right\|_{H_{0}^{1}}$ and $\|z(t)\|_{H_{0}^{1}} \leq 2 e^{-\beta t}\left\|z_{0}\right\|_{H_{0}^{1}}$.

Remark 2.8. Rankin [29] considered well-posedness properties for a certain type of semilinear evolution equations where the nonlinear terms are in divergence form. According to his results, we can see that equation (2.3) has a unique (strong) solution for initial data in $L^{p}(0, \ell), p \geq 4$. To get this result, he used the analyticity of the semigroup $S(t)$ and the fact 
that the differential operator $\frac{d}{d x}$ on $H_{0}^{1}(0, \ell)$ can be represented by

$$
\sqrt{\epsilon} \frac{d}{d x}=\left(-A_{\varepsilon}\right)^{\frac{1}{2}} B
$$

for some bounded operator $B \in \mathcal{L}(H)$, where $H=L^{2}(0, \ell)$. In general, it is not true that $\sqrt{\epsilon} \frac{d}{d x}=\left(-A_{\epsilon}\right)^{\frac{1}{2}}$. This result could be used to analyze the stability property of the solution of Burgers' equation with initial data in $L^{p}(0, \ell), p \geq 4$.

\section{Linear control problem}

As we noted in Section 2, the open-loop (no control) solution of Burgers' equation (2.3) decays exponentially in the topology of the energy space $H_{0}^{1}(0, \ell)$ (see Theorem 2.7). However, the decay rate depends on the viscosity $\epsilon>0$. We now explore the possibility of obtaining an exponential decay rate independent of viscosity by feedback laws.

The basic model is governed by an abstract system of the form

$$
\begin{aligned}
\frac{d}{d t} z(t) & =A z(t)+B u(t), \quad z(0)=z_{0} \in H, \\
y(t) & =C z(t), \quad t \geq 0,
\end{aligned}
$$

where $H, U$ and $Y$ are Hilbert spaces, $u(\cdot) \in L^{2}(0, \infty ; U), y(\cdot) \in L^{2}(0, \infty ; Y)$, and $A$ is the infinitesimal generator of an analytic semigroup $S(t)$ on a Hilbert space $H$. Assume that $B \in \mathcal{L}(U, H), C \in \mathcal{L}(H, Y)$ and $A$ is self-adjoint with compact resolvent. Solutions of (3.1) are taken to be in mild form, i.e.,

$$
\begin{aligned}
& z(t)=S(t) z_{0}+\int_{0}^{t} S(t-s) B u(s) d s, \\
& y(t)=C S(t) z_{0}+C \int_{0}^{t} S(t-s) B u(s) d s, \quad t \geq 0 .
\end{aligned}
$$

We first consider the performance index

$$
J(u)=\int_{0}^{\infty}\left\{\|y(t)\|_{Y}^{2}+R\|u(t)\|_{U}^{2}\right\} d t
$$

where $y(t)$ is given by equation (3.3), and $R>0$. The linear quadratic regulator problem is (LQR) : Find $u(\cdot) \in L^{2}(0, \infty ; U)$ minimizing the cost functional $J$ given by equation 
(3.4) subject to the system (3.2)-(3.3).

For the existence of an admissible control $u$ such that $J(u)<\infty$ and for the exponential stability of the closed-loop system we need the following two hypotheses.

(H1). The system (3.1) is stabilizable in the sense that there is a feedback operator $K \in \mathcal{L}(H, U)$ such that the closed loop semigroup $S_{K}(t) \in \mathcal{L}(H)$ given by

$$
S_{K}(t) z=S(t) z+\int_{0}^{t} S(t-s) B K S_{K}(s) z d s
$$

for all $t \geq 0$ and $z \in H$ decays exponentially.

(H2). The system (3.1) is detectable in the sense that there exists an operator $F \in$ $\mathcal{L}(Y, H)$ such that the output injection semigroup $S_{F}(t) \in \mathcal{L}(H)$ given by

$$
S_{F}(t) z=S(t) z+\int_{0}^{t} S_{F}(t-s) F C S(s) z d s
$$

for all $t \geq 0$ and $z \in H$ decays exponentially.

Remark 3.1.[28, pp. 134-135] (i) If (H1) is satisfied, then for any $z_{0} \in H$, there is an admissible control $u_{z_{0}}(\cdot) \in L^{2}(0, \infty ; U)$ such that $J\left(u_{z_{0}}\right)<\infty$.

(ii) Let (H2) be satisfied. Then for any $z_{0} \in H$ and $u(\cdot) \in L^{2}(0, \infty ; U)$ with $J(u)<\infty$, $z(t)$ defined by equation $(3.2)$ is in $L^{2}(0, \infty ; H)$.

Now we state the following fundamental results for (LQR) problem, see $[4,13]$.

Theorem 3.2. Let hypotheses $(\mathrm{H} 1)$ and $(\mathrm{H} 2)$ be satisfied. Then there is a unique optimal control $\bar{u}(\cdot) \in L^{2}(0, \infty ; U)$ for the linear quadratic regulator (LQR) problem and $\bar{u}(\cdot)$ is given by the feedback law

$$
\bar{u}(t)=-R^{-1} B^{*} \Pi \bar{z}(t), \quad t \geq 0
$$

where $\bar{z}(t)$ is the corresponding optimal trajectory and $\Pi \in \mathcal{L}(H)$ is the unique nonnegative self-adjoint solution of the algebraic Riccati equation

$$
A^{*} \Pi z+\Pi A z-\Pi B R^{-1} B^{*} \Pi z+C^{*} C z=0
$$

for every $z \in \mathcal{D}(A)$. Moreover, the closed-loop semigroup $S_{\Pi}(t) \in \mathcal{L}(H)$ generated by the operator $A-B R^{-1} B^{*} \Pi$ decays exponentially. 
Remark 3.3. The algebraic Riccati equation (3.8) can be defined for all $z \in H$, since the right hand side of equation

$$
\Pi A z=-A^{*} \Pi z+\Pi B R^{-1} B^{*} \Pi z-C^{*} C z
$$

is well-defined for all $z \in H, \mathcal{D}(A)$ is densely embedded in $H$, and hence we can extend the left hand side of equation (3.9) to $z \in H$ continuously.

We now consider the control problem, and its linearization, for Burgers' equation. Define the operator $A_{\epsilon}$, as in Section 2, by $A_{\epsilon} \phi=\epsilon \phi^{\prime \prime}$ for all $\phi \in \mathcal{D}\left(A_{\epsilon}\right)=H^{2}(0, \ell) \cap H_{0}^{1}(0, \ell)$. For the control input operator $B$ and the observation output operator $C$ we consider the Hilbert spaces $H=L^{2}(0, \ell), U=\mathbf{R}$ and $Y=\mathbf{R}^{k}$. Assume that $B \in \mathcal{L}(U, H)$ and $C \in \mathcal{L}(H, Y)$ are defined by

$$
B u=b(\cdot) u \quad \text { and } \quad C z=\left(\tilde{z}\left(\bar{x}_{1}\right), \cdots, \tilde{z}\left(\bar{x}_{k}\right)\right),
$$

where $b(\cdot) \in H, u \in U$, and $\bar{x}_{i} \in(0, \ell), 1 \leq i \leq k$, are defined by

$$
\tilde{z}\left(\bar{x}_{i}\right)=\frac{1}{2 \delta} \int_{\bar{x}_{i}-\delta}^{\bar{x}_{i}+\delta} z(x) d x .
$$

In equation $(3.11), \delta>0$ is chosen so that $\left(\bar{x}_{i}-\delta, \bar{x}_{i}+\delta\right) \subset(0, \ell)$ for all $1 \leq i \leq k$.

Consider the following linear control problem.

$(\mathrm{LQR})_{\alpha}:$ Find $\bar{u}(\cdot) \in L^{2}(0, \infty ; U)$ minimizing the weighted performance index

$$
J(u)=\int_{0}^{\infty}\left\{\|y(t)\|_{Y}^{2}+R\|u(t)\|_{U}^{2}\right\} e^{2 \alpha t} d t, \quad(\alpha>0)
$$

subject to the governing equations

$$
\begin{aligned}
\frac{d}{d t} z(t) & =A_{\varepsilon} z(t)+B u(t), \quad z(0)=z_{0} \\
y(t) & =C z(t), \quad t \geq 0,
\end{aligned}
$$

where $A_{\epsilon}, B, C$ are as above and $R>0$.

Remark 3.4. (i) Equation (3.13) corresponds to the system

$$
\begin{aligned}
\frac{\partial}{\partial t} z(t, x) & =\epsilon \frac{\partial^{2}}{\partial x^{2}} z(t, x)+b(x) u(t), \quad t>0, \quad 0<x<\ell \\
v(t, 0) & =v(t, \ell)=0 \\
v(0, x) & =v_{0}(x)
\end{aligned}
$$


(ii) For each $i, 1 \leq i \leq k, \tilde{z}\left(\bar{x}_{i}\right)$ given by equation (3.11) represents an average value of $z(x)$ over a small neighborhood of $\bar{x}_{i}$. We can regard each $\bar{x}_{i}, 1 \leq i \leq k$, as the location of a "weight".

(iii) The weight function $e^{2 \alpha t}$ in the definition (3.12) of the cost functional $J$ will play an important role in the exponential decay rate, see Theorems 3.9 and 3.10. However, it also gives rise to the question of existence of an admissible control $u(\cdot)$ such that $J(u)<\infty$.

For the control problem (LQR) $)_{\alpha}$, we introduce an " $\alpha$-shifted" control system [15]. Let $\hat{z}(t)=z(t) e^{\alpha t}, \hat{u}(t)=u(t) e^{\alpha t}$ and $\hat{y}(t)=y(t) e^{\alpha t}$. We then have a modified linear control problem

$(\mathrm{LQR})_{\hat{\alpha}}:$ Find $\overline{\hat{u}} \in L^{2}(0, \infty ; U)$ minimizing the cost functional

$$
\hat{J}(\hat{u})=\int_{0}^{\infty}\left\{\|\hat{y}(t)\|_{Y}^{2}+R\|\hat{u}(t)\|_{U}^{2}\right\} d t
$$

subject to

$$
\begin{aligned}
\frac{d}{d t} \hat{z}(t) & =\left(A_{\epsilon}+\alpha I\right) \hat{z}(t)+B \hat{u}(t), \quad \hat{z}(0)=z_{0}, \\
\hat{y}(t) & =C \hat{z}(t), \quad t \geq 0 .
\end{aligned}
$$

The solutions for the system (3.17) again are taken as mild solutions. If we solve the problem (LQR) $\hat{\alpha}$ and apply

$$
\bar{u}_{\alpha}(t)=e^{-\alpha t} \overline{\hat{u}}(t) \quad(t \geq 0)
$$

to the original control system $(3.13)-(3.14)$, then the resulting optimal trajectory $\bar{z}_{\alpha}(t)$ will satisfy the inequality

$$
\left\|\bar{z}_{\alpha}(t)\right\|_{H} \leq M e^{-\alpha t}\left\|z_{0}\right\|_{H}
$$

where $M \geq 1$ is a constant and $\alpha>0$ is the desired degree of stability.

Remark 3.5. A discussion of the " $\alpha$-shifted" problem for finite dimensional systems first appeared in [1]. Anderson and Moore showed that, for finite dimensional systems, the control problem (LQR $)_{\alpha}$ is "equivalent" to $(\mathrm{LQR})_{\alpha} \hat{\alpha}$ in the following senses :

(i) The minimum value of $J$ defined by equation (3.12) is the same as the minimum value of $\hat{J}$ given by equation (3.16). 
(ii) If $\overline{\hat{u}}(t)=g(\hat{z}(t))$ is the optimal control for (LQR) $\hat{\alpha}$ for some function $g$, then $\bar{u}(t)=$ $e^{-\alpha t} g\left(z(t) e^{\alpha t}\right)$

is the optimal control for (LQR) $)_{\alpha}$ and conversely.

We now apply Theorem 3.2 to the problem (LQR) $\hat{\alpha}$ in order to obtain an optimal control $\overline{\hat{u}} \in L^{2}(0, \infty ; U)$ for $(\mathrm{LQR}) \hat{\alpha}$.

Remark 3.6. (i) From Remark 2.2, the spectrum $\sigma\left(A_{\epsilon}+\alpha I\right)$ of the infinitesimal generator $A_{\epsilon}+\alpha I$ consists of all eigenvalues $\lambda_{\alpha, n}, n=1,2, \cdots$, given by $\lambda_{\alpha, n}=\alpha-\epsilon n^{2} \pi^{2} / \ell^{2}$ and for each $n, n=1,2, \cdots$, the eigenfunction $\phi_{\alpha, n}$ corresponding to $\lambda_{\alpha, n}$ is given by $\phi_{\alpha, n}(x)=\sqrt{2} \sin \frac{n \pi}{\ell} x$.

(ii) We are interested in the stabilization problem for the system (3.15) with small viscosity $\epsilon=\frac{1}{\mathrm{Re}}>0$, i.e., high Reynolds number. Let $\alpha_{0}=\frac{\epsilon \pi^{2}}{\ell^{2}}$. If $\alpha>\alpha_{0}$, then there is at least one positive eigenvalue $\lambda_{\alpha, 1}=\alpha-\frac{\epsilon \pi^{2}}{\ell^{2}}$. Moreover, if $\alpha>\alpha_{0}$ and $0<\gamma \leq \epsilon$, then the first eigenvalue $\alpha-\frac{\gamma \pi^{2}}{\ell^{2}}$ of $A_{\gamma}+\alpha I$ satisfies $\alpha-\frac{\gamma \pi^{2}}{\ell^{2}}>\alpha-\frac{\epsilon \pi^{2}}{\ell^{2}}>0$ and hence $A_{\gamma}+\alpha I$ will have at least one positive eigenvalue for all $0<\gamma \leq \epsilon$. This will become important when we apply feedback laws computed from low Reynolds number to investigate the closed-loop response of the nonlinear Burgers' equation for high Reynolds numbers (see Example 3 in Section 4).

Let $\alpha>\alpha_{0}=\frac{e \pi^{2}}{\ell^{2}}$ be given and let

$$
n_{\alpha}=\max \left\{n \in \mathbf{N}: \lambda_{\alpha, n}=\alpha-\frac{\epsilon n^{2} \pi^{2}}{\ell^{2}} \geq 0\right\} .
$$

Since $A_{\epsilon}$ is self-adjoint (see Remark 2.2) and the set $\left\{\phi_{\alpha, n}: n=1,2, \cdots\right\}$ is a basis for $H=L^{2}(0, \ell)$, we can identify $z \in H$ with the sequence $\left\{<z, \phi_{\alpha, n}>\right\}_{n \in \mathbb{N}}$. Assume that $b_{n} \in U$ and $c_{n} \in Y$ satisfy

$$
B u=\left\{\left\langle b_{n}, u>\right\}_{n \in \mathbf{N}} \quad \text { and } \quad C z=\sum_{n=1}^{\infty} c_{n}<z, \phi_{\alpha, n}>\right.
$$

with $\sum_{n=1}^{\infty}\left\|b_{n}\right\|_{U}^{2}<\infty$ and $\sum_{n=1}^{\infty}\left\|c_{n}\right\|_{Y}^{2}<\infty$, see [28, pp. 137-143].

The following lemma is an application of stabilizability and detectability results of Pritchard and Salamon [28, Section 4.2].

Lemma 3.7. For each $n=1,2, \cdots, n_{\alpha}$, let

$$
X_{\alpha, n}=\left\{\frac{i \ell}{n}: i=1,2, \cdots, n-1\right\} \text {. }
$$


Then the following statements hold.

(a) $b_{n}=<b(\cdot), \phi_{\alpha, n}>\neq 0$ for all $n=1,2, \cdots, n_{\alpha}$, if and only if the system (3.17) is stabilizable in $H$.

(b) If $\delta>0$ satisfies $\delta<\frac{\ell}{2 n_{\alpha}}$, then for each $n=1,2, \cdots, n_{\alpha}$, there exists at least one $\bar{x}_{i}, 1 \leq i \leq k$, such that $\bar{x}_{i} \notin X_{\alpha, n}$ if and only if the system (3.17) is detectable through $C \in \mathcal{L}(H, Y)$.

Proof: (a) From Remark 3.6, we know that the spectrum $\sigma\left(A_{\epsilon}+\alpha I\right)$ of $A_{\epsilon}+\alpha I$ consists of all eigenvalues $\lambda_{\alpha, n}=\alpha-\epsilon n^{2} \pi^{2} / \ell^{2}$. Thus, for all $n, n \geq n_{\alpha}+1$, we know that $\lambda_{\alpha, n}<0$. Let $H_{u}$ be the linear span of eigenfunctions $\phi_{\alpha, 1}, \cdots, \phi_{\alpha, n_{\alpha}}$. Then the dimension of $H_{u}$ is $n_{\alpha}$ and hence the system (3.17) is stabilizable if and only if the projection of (3.17) onto $H_{u}$ is controllable, or equivalently, if and only if $b_{n}=<b(\cdot), \phi_{\alpha, n}>\neq 0$ for all $n, n=1,2, \cdots, n_{\alpha}$.

For $(\mathrm{b})$, let $c_{n}=\left(c_{n 1}, \cdots, c_{n k}\right), n=1,2, \cdots$, be defined by equation (3.21). Then we have

$$
c_{n i}=\frac{\sqrt{2} \ell}{n \pi \delta} \sin \frac{n \pi \bar{x}_{i}}{\ell} \sin \frac{n \pi \delta}{\ell}
$$

for $1 \leq i \leq k$. By the dual statements of (a), the system (3.17) is detectable through $C \in \mathcal{L}(H, Y)$ if and only if $c_{n} \neq 0$ for all $n=1,2, \cdots, n_{\alpha}$. Hence, (b) holds.

Remark 3.8. If $n_{\alpha}=1$, then $X_{\alpha, 1}$ is the empty set.

We now return to the original control problem $(\mathrm{LQR})_{\alpha}$. The following theorem is the main result for our control problem (LQR) $)_{\alpha}$.

Theorem 3.9. Let $\alpha>\alpha_{0}$ be given. Suppose that $b(\cdot) \in H=L^{2}(0, \ell), \delta>0, \bar{x}_{i}, 1 \leq i \leq$ $k$, satisfy the conditions (a) and (b) of Lemma 3.7. Then there is a unique optimal control $\bar{u}_{\alpha, \epsilon}(\cdot) \in L^{2}(0, \infty ; \mathbf{R})$ for the problem (LQR) $)_{\alpha}$ such that

$$
\bar{u}_{\alpha, \varepsilon}(t)=-R^{-1} B^{*} \Pi_{\alpha, \varepsilon} \bar{z}_{\alpha, \epsilon}(t), \quad t \geq 0,
$$

where $\bar{z}_{\alpha, \epsilon}(t)$ is the corresponding optimal trajectory and $\Pi_{\alpha, \epsilon} \in \mathcal{L}(H)$ is the unique nonnegative self-adjoint operator satisfying the algebraic Riccati equation

$$
\left(A_{\epsilon}+\alpha I\right)^{*} \Pi_{\alpha, \epsilon} z+\Pi_{\alpha, \epsilon}\left(A_{\epsilon}+\alpha I\right) z-\Pi_{\alpha, \epsilon} B R^{-1} B^{*} \Pi_{\alpha, \epsilon} z+C^{*} C z=0
$$

for every $z \in \mathcal{D}\left(A_{\epsilon}\right)=H^{2}(0, \ell) \cap H_{0}^{1}(0, \ell)$. Moreover, the closed loop semigroup $S_{\Pi_{a, \epsilon}}(t) \in$ $\mathcal{L}(H)$ satisfies the following stability property

$$
\left\|S_{\Pi_{\alpha, \varepsilon}}(t)\right\|_{\mathcal{L}(H)} \leq M e^{-(\alpha+\omega) t}
$$


for some constants $M=M(\alpha, \epsilon) \geq 1$ and $\omega=\omega(\alpha, \epsilon)>0$.

Proof: By Lemma 3.7, we know that the $\alpha$-shifted control system (3.17) satisfies all hypotheses (H1) and (H2) (with $z(t), y(t), u(t), A, S(t)$ and $J$ replaced by $\hat{z}(t), \hat{y}(t), \hat{u}(t)$, $A_{\epsilon}+\alpha I, \hat{S}(t)$ and $\hat{J}$, respectively ). Hence, by Theorem 3.2 , there is a unique optimal control $\overline{\hat{u}}(t)$ for (LQR) $)_{\alpha}$ and the corresponding closed-loop semigroup $\hat{S}(t)$ decays exponentially, i.e.,

$$
\|\hat{S}(t)\|_{\mathcal{L}(H)} \leq \hat{M} e^{-\omega t} \quad t \geq 0
$$

for some constants $\hat{M}=\hat{M}(\alpha, \epsilon) \geq 1$ and $\omega=\omega(\alpha, \epsilon)>0$. Moreover, $\overline{\hat{u}}(t)$ is given by

$$
\overline{\hat{u}}(t)=-R^{-1} B^{*} \Pi_{\alpha, c} \overline{\hat{z}}(t)
$$

where $\overline{\hat{z}}(t)$ is the resulting optimal trajectory for the $\alpha$-shifted system $(3.17)$ and $\Pi_{\alpha, \epsilon}$ is the unique nonnegative self-adjoint solution of equation (3.25). Since the semigroup $\hat{S}(t)$ is generated by $A_{\epsilon}+\alpha I-B R^{-1} B^{*} \Pi_{\alpha, \epsilon}$, the infinitesimal generator of the closed-loop semigroup $S_{\Pi_{\alpha, e}}(t)$ for the original system (3.13) is $A_{\epsilon}-B R^{-1} B^{*} \Pi_{\alpha, \epsilon}$. Hence, $S_{\Pi_{\alpha, \epsilon}}(t)=\hat{S}(t) e^{-\alpha t}$ and, by the relation (3.27), $S_{\Pi_{\alpha, \epsilon}}(t)$ satisfies the inequality (3.26) with $M=\hat{M}$. Moreover, the optimal control $\bar{u}_{\alpha, \epsilon}(t)$ for $(\mathrm{LQR})_{\alpha}$ is given by the formula $(3.24)$, since $\bar{u}_{\alpha, \epsilon}(t)=\overline{\hat{u}}(t) e^{-\alpha t}=$ $-R^{-1} B^{*} \Pi_{\alpha, \epsilon} \overline{\hat{z}}(t) e^{-\alpha t}=-R^{-1} B^{*} \Pi_{\alpha, c} \bar{z}_{\alpha, c}(t)$, where $\bar{z}_{\alpha, c}(t)=\overline{\hat{z}}(t) e^{-\alpha t}$ is the corresponding optimal trajectory for the original system (3.13). This completes the proof.

The optimal control $\bar{u}_{\alpha, \epsilon}(\cdot) \in L^{2}(0, \infty ; \mathbf{R})$ obtained in Theorem 3.9 is given by the feedback law (3.24). Note that $\bar{u}_{\alpha, \epsilon}(t)$ depends on $\alpha$ and $\epsilon$. Define the feedback operator $K_{\alpha, \varepsilon} \in \mathcal{L}(H, U)$ by

$$
K_{\alpha, \epsilon}=-R^{-1} B^{*} \Pi_{\alpha, \epsilon}
$$

Then the optimal control $\bar{u}_{\alpha, \epsilon}(t), t \geq 0$, is given by

$$
\bar{u}_{\alpha, \epsilon}(t)=K_{\alpha, \varepsilon} \bar{z}_{\alpha, \varepsilon}(t)
$$

and the infinitesimal generator for the closed-loop semigroup $S_{\Pi_{\alpha, \epsilon}}(t)$ is

$$
A_{\epsilon}+B K_{\alpha, \epsilon}=A_{\epsilon}-B R^{-1} B^{*} \Pi_{\alpha, \epsilon} \text {. }
$$

Recall that $H=L^{2}(0, \ell)$ and $U=\mathbf{R}$. Thus, by Riesz representation theorem (see, e.g., $\left[11\right.$, p. 13]), there is a unique feedback gain function $k_{\alpha, \epsilon}(\cdot) \in L^{2}(0, \ell)$ such that

$$
K_{\alpha, c} z=\int_{0}^{\ell} k_{\alpha, c}(s) z(s) d s
$$


for all $z \in L^{2}(0, \ell)$.

We now have the following main result of this section.

Theorem 3.10. Let $\alpha>\alpha_{0}$ be given. Suppose that $b(\cdot) \in H=L^{2}(0, \ell), \delta>0$ and $\bar{x}_{i}, 1 \leq i \leq k$, satisfy the conditions (a) and (b) of Lemma 3.7. Let $k_{\alpha, \epsilon}(\cdot) \in H$ be the linear feedback gain function defined by the formula (3.32). Then there exist constants $\rho=\rho(\alpha, \epsilon)>0$ and $M=M(\alpha, \epsilon) \geq 1$ such that for any initial data $z_{0}(\cdot) \in H_{0}^{1}(0, \ell)$, with $\left\|z_{0}\right\|_{H_{0}^{1}} \leq \frac{\rho}{2 M}$, the controlled Burgers' equation

$$
\begin{aligned}
\frac{\partial}{\partial t} z(t, x) & =\epsilon \frac{\partial^{2}}{\partial x^{2}} z(t, x)-z(t, x) \frac{\partial}{\partial x} z(t, x)+b(x) \int_{0}^{\ell} k_{\alpha, \epsilon}(s) z(t, s) d s \\
z(t, 0) & =z(t, \ell)=0 \\
z(0, x) & =z_{0}(x) \in H_{0}^{1}(0, \ell)
\end{aligned}
$$

has a unique (strong) solution and the solution $z(t)(\cdot)=z(t, \cdot)$ satisfies the following stability property

$$
\|z(t)\|_{H_{0}^{1}} \leq 2 M e^{-\alpha t}\left\|z_{0}(\cdot)\right\|_{H_{0}^{1}}
$$

Proof: Let the operators $A_{\epsilon}, B, C$ and $K_{\alpha, \epsilon}$ be given by equations (2.4), (3.10) and (3.29). Define the nonlinear function $f: H_{0}^{1}(0, \ell) \rightarrow L^{2}(0, \ell)$ by

$$
f(z)=B K_{\alpha, \epsilon} z-z z^{\prime}
$$

where ${ }^{\prime}=\frac{d}{d x}$. Then, the map $f$ satisfies the hypothesis $(\mathrm{F})$ in Section 2 , since for any $z_{1}, z_{2} \in H_{0}^{1}(0, \ell)$,

$$
\left\|f\left(z_{1}\right)-f\left(z_{2}\right)\right\|_{L^{2}(0, \ell)} \leq\left(\left\|B K_{\alpha, \epsilon}\right\|_{\mathcal{C}(H)}+\left\|z_{1}\right\|_{H_{0}^{1}}+\left\|z_{2}\right\|_{H_{0}^{1}}\right)\left\|z_{1}-z_{2}\right\|_{H_{0}^{1}}
$$

Note that the operator $B K_{\alpha, \epsilon}$ is bounded on the state space $H=L^{2}(0, \ell)$. Thus, by Theorem 2.1 , we have a unique local (strong) solution of equation (3.33).

Let $S_{K_{\alpha, c}}(t), t \geq 0$, be the analytic semigroup on $H$ generated by the operator $A_{\epsilon}+$ $B K_{\alpha, \epsilon}$. Then, by Theorem $3.9, S_{K_{\alpha, \epsilon}}(t)$ satisfies the inequality

$$
\left\|S_{K_{\alpha, \epsilon}}(t) z\right\|_{H} \leq M_{\alpha} e^{-(\alpha+\omega) t}\|z\|_{H}
$$


for all $z \in H$ and for some constants $M_{\alpha}=M_{\alpha}(\alpha, \epsilon) \geq 1$ and $\omega=\omega(\alpha, \epsilon)>0$. Let $\alpha<\beta<\alpha+\omega$. Then, there is a constant $\tilde{M}_{\alpha}=\tilde{M}_{\alpha}(\alpha, \epsilon, \beta) \geq 1$ such that

$$
\begin{aligned}
\left\|S_{K_{\alpha, \epsilon}}(t) z\right\|_{H_{0}^{1}} & \leq M_{\alpha} e^{-\beta t}\|z\|_{H_{0}^{1}} \\
\left\|S_{K_{\alpha, \epsilon}}(t) z\right\|_{H_{0}^{1}} & \leq \tilde{M}_{\alpha} \frac{1}{\sqrt{t}} e^{-\beta t}\|z\|_{H}
\end{aligned}
$$

for all $z \in H_{0}^{1}$. Let $M=\max \left\{M_{\alpha}, \tilde{M}_{\alpha}\right\}$ and choose $\rho>0$ with $0<\rho<\frac{\sqrt{\beta-\alpha}}{2 \sqrt{\pi} M}$. Then it is easy to see that

$$
\rho M \int_{0}^{\infty} \frac{1}{\sqrt{s}} e^{-(\beta-\alpha) s} d s<\frac{1}{2} .
$$

Thus, by arguments similar to those in the proof of Theorem 2.7 together with inequalities (3.38)-(3.39) and the expression

$$
z(t)=S_{K_{\alpha, e}}(t) z_{0}+\int_{0}^{t} S_{K_{\alpha, e}}(t-s) g(z(s)) d s
$$

the unique global solution $z(t)(\cdot)=z(t, \cdot)$ for the controlled Burgers' equation (3.33) exists and satisfies the inequality (3.34), where $g(z(t))=-z(t) z^{\prime}(t)$.

\section{Approximation and numerical results}

In Section 3, we considered a linear quadratic regulator problem (LQR) $)_{\alpha}$ to obtain a desired degree of stability for the solution of the closed-loop Burgers' equation. In this section we consider an approximation scheme for $(\mathrm{LQR})_{\alpha}$ and give some numerical results.

We first introduce an abstract approximation scheme for the problem (LQR) based on the results of Banks, Kunish [4] and Ito [19] and then apply the scheme to get the optimal control $\bar{u}_{\alpha, \varepsilon}(\cdot) \in L^{2}(0, \infty ; \mathbf{R})$. Throughout this section, we assume that $R=I$, the identity operator on the control space $U$, (see Section 3 ). For more approximation schemes for the linear regulator problems, see $[2,4,14]$ and references given there.

We formulate a sequence of approximate regulator problems and present a convergence result for the corresponding Riccati operators. Throughout this section, we use superscript $N$ in the designation of subspaces, operators and matrices in the $N$-th approximating system and control problem, like $H^{N}, A^{N}, B^{N}$, etc. Hence the superscript $N$ indicates the order of approximation. 
Let $H^{N}, N=1,2, \cdots$, be a sequence of finite dimensional linear subspaces of $H$ and $P^{N}: H \rightarrow H^{N}$ be the canonical orthogonal projections. Assume that $S^{N}(t)$ is a sequence of $C_{0}$-semigroups on $H^{N}$ with infinitesimal generators $A^{N} \in \mathcal{L}\left(H^{N}\right)$. We then consider the family of regulator problems:

$(\mathrm{LQR})^{N}:$ Minimize $J^{N}\left(z_{0}^{N}, u\right)$ over $u \in L^{2}(0, \infty ; U)$ subject to the control system

$$
\begin{aligned}
& z^{N}(t)=S^{N}(t) z_{0}^{N}+\int_{0}^{t} S^{N}(t-s) B^{N} u(s) d s, \\
& y^{N}(t)=C^{N} z^{N}(t), \\
& \text { where } z^{N}(0)=z_{0}^{N} \equiv P^{N} z_{0} \text { and } \\
& J^{N}\left(z_{0}^{N}, u\right)=\int_{0}^{\infty}\left\{\left\|y^{N}(t)\right\|_{Y}^{2}+\|u(t)\|_{U}^{2}\right\} d t .
\end{aligned}
$$

Remark 4.1. If, for each $N,\left(A^{N}, B^{N}\right)$ is stabilizable and $\left(A^{N}, C^{N}\right)$ is detectable, then, by Theorem 3.2 , there is a unique optimal control $\bar{u}^{N}(t)$ for the finite dimensional problem $(\mathrm{LQR})^{N}$ and it is given by

$$
\bar{u}^{N}(t)=-\left(B^{N}\right)^{*} \Pi^{N} S_{\Pi}^{N}(t) z_{0}^{N}
$$

where $S_{\Pi}^{N}(t)$ is the $C_{0}$-semigroup on $H^{N}$ generated by $A^{N}-B^{N}\left(B^{N}\right)^{*} \Pi^{N}$ and $\Pi^{N} \in \mathcal{L}\left(H^{N}\right)$ is the unique nonnegative self-adjoint solution of

$$
\left(A^{N}\right)^{*} \Pi^{N}+\Pi^{N} A^{N}-\Pi^{N} B^{N}\left(B^{N}\right)^{*} \Pi^{N}+\left(C^{N}\right)^{*} C^{N}=0 .
$$

For the finite dimensional approximation systems, it is not clear that $\left(A^{N}, B^{N}\right)$ is stabilizable even if the original system $(A, B)$ is stabilizable. Similarly, it is not clear that the detectability property of $(A, C)$ is preserved under the finite dimensional projections. Another question we have to consider is the convergence of approximates $\Pi^{N}$ and $\bar{u}^{N}(t)$ to the infinite dimensional solutions $\Pi$ and $\bar{u}(t)$, respectively. For these reasons, we need the following assumptions.

Let $S^{N}(t)=e^{A^{N} t}, t \geq 0$.

$(A 1)$ : For each $z \in H, S^{N}(t) P^{N} z \longrightarrow S(t) z$ and $S^{N}(t)^{*} P^{N} z \longrightarrow S(t)^{*} z$, where the convergences are uniform in $t$ on bounded subsets of $[0, \infty)$.

(A2): (i) For each $u \in U, B^{N} u \longrightarrow B u$ and for each $z \in H,\left(B^{N}\right)^{*} P^{N} z \longrightarrow B^{*} z$. 
(ii) For each $z \in H, C^{N} P^{N} z \longrightarrow C z$ and for each $y \in Y,\left(C^{N}\right)^{*} y \longrightarrow C^{*} y$.

(A3): (i) The family of systems $\left(A^{N}, B^{N}\right)$ is uniformly stabilizable, i.e., there exists a sequence of operators $K^{N} \in \mathcal{L}\left(H^{N}, U\right)$ such that sup $\left\|K^{N}\right\|<\infty$ and

$$
\left\|e^{\left(A^{N}+B^{N} K^{N}\right) t} P^{N}\right\|_{\mathcal{L}(H)} \leq M_{1} e^{-\omega_{1} t}, \quad t \geq 0,
$$

for some positive constants $M_{1} \geq 1$ and $\omega_{1}>0$ which are independent of $N$.

(ii) The family of pairs $\left(A^{N}, C^{N}\right)$ is uniformly detectable, i.e., there exists a sequence of operators $F^{N} \in \mathcal{L}\left(Y, H^{N}\right)$ such that sup $\left\|F^{N}\right\|<\infty$ and

$$
\left\|e^{\left(A^{N}+F^{N} C^{N}\right) t} P^{N}\right\|_{\mathcal{L}(H)} \leq M_{2} e^{-\omega_{2} t}, \quad t \geq 0
$$

for some constants $M_{2} \geq 1$ and $\omega_{2}>0$ which are independent of $N$.

Remark 4.2. (i) The condition (A3)(ii) is a relaxation of the coercivity assumption in [4] (see also $[19$, p.3]).

(ii) Suppose that $B^{N}=P^{N} B$ and $C^{N}=C P^{N}$. Then (A2) holds, since it follows from (A1) that $P^{N} z \rightarrow z$ for all $z \in H$.

By simple modification of results from [19, Theorem 2.1] and [4, Theorem 2.2], we have the following fundamental convergence results.

Theorem 4.3. Let $(A, B)$ be stabilizable and $(A, C)$ be detectable. Suppose that $(A 1)-$ (A3) are satisfied. Then, for each $N$, the finite dimensional algebraic Riccati Equation (4.5) admits a uique nonnegative self-adjoint solution $\Pi^{N}$ such that $\sup \left\{\left\|\Pi^{N}\right\|_{\mathcal{L}\left(H^{N}\right)}: N=\right.$ $1,2, \cdots\}<\infty$ and

$$
\Pi^{N} P^{N} z \longrightarrow \Pi z
$$

for every $z \in H$. Moreover, there exist positive constants $M_{3} \geq 1$ and $\omega_{3}$ (independent of $N$ ) such that

$$
\left\|e^{\left(A^{N}-B^{N}\left(B^{N}\right)^{*} \Pi^{N}\right) t} P^{N}\right\|_{\mathcal{L}(H)} \leq M_{3} e^{-\omega_{3} t}, \quad t \geq 0 .
$$

For the uniform stabilizability and detectability assumptions $(A 3)$ we introduce a sesquilinear form $a_{\epsilon}(\cdot, \cdot): V \times V \rightarrow \mathbf{C}$ defined by

$$
a_{\epsilon}(z, w)=\int_{0}^{1} \epsilon z^{\prime}(x) \bar{w}^{\prime}(x) d x, \quad z, w \in V
$$


where $V=H_{0}^{1}(0,1)$. Note that, to allow the use of the theory of sectorial operators and sesquilinear forms in discussing the spectra of various operators, we assume in defining $a_{\varepsilon}(\cdot, \cdot)$ that the functions in $V$ are complex valued. It is easy to see that the sesquilinear form $a_{\epsilon}(\cdot, \cdot)$ is $V$-coercive [36, p. 274], i.e.,

$$
\begin{aligned}
& \left|a_{\epsilon}(z, w)\right| \leq \epsilon\|z\|_{V}\|w\|_{V}, \quad \text { (continuity), } \\
& \mathcal{R} \text { e } a_{\epsilon}(z, z)+\gamma\|z\|_{H}^{2} \geq \epsilon\|z\|_{V}^{2}, \quad \text { (Gårding's inequality), }
\end{aligned}
$$

for all $z, w \in V$ and $\gamma \geq \epsilon>0$. Furthermore, it follows from the bounds (4.9) and (4.10) that there exists, in a unique manner, an operator $A_{\varepsilon} \in \mathcal{L}\left(V, V^{*}\right)$ such that

$$
a_{\epsilon}(z, w)=<-A_{\epsilon} z, w>_{V^{*}, V} \quad \text { and } \quad \overline{a_{\epsilon}(z, w)}=<-A_{\epsilon}^{*} w, z>_{V^{*}, V}
$$

for all $z, w \in V$ (see, e.g., [36, pp. 271-275]).

Turning next to specific approximations for $(L Q R)_{\alpha}$, we divide the unit interval $[0,1]$ into $N+1$ equal subintervals to get $\left[x_{i}, x_{i+1}\right], x_{i}=\frac{i}{N+1}, i=0,1, \cdots, N$. For each $i, 1 \leq i \leq N$, let $h_{i}^{N}(x)$ denote the linear spline basis function defined by

$$
h_{i}^{N}(x)= \begin{cases}(N+1)\left(x-x_{i-1}\right), & x_{i-1} \leq x \leq x_{i} \\ -(N+1)\left(x-x_{i+1}\right), & x_{i} \leq x \leq x_{i+1} \\ 0, & \text { otherwise }\end{cases}
$$

Let $H^{N}$ be the $N$-dimensional finite element space given by

$$
H^{N}=\left\{\sum_{i=1}^{N} z_{i} h_{i}^{N}(x): z_{i} \in \mathbf{R}, i=1,2, \cdots, N\right\} .
$$

Then we have a sequence of finite dimensional (real) subspaces $H^{N} \subset V, n=1,2, \cdots$. Moreover, it is well-known [31],[19, p. 15] that the family of $H^{N}$ satisfies the following approximation condition:

(APP) : For each $z \in V$, there exists an element $z^{N} \in H^{N}$ such that

$$
\left\|z-z^{N}\right\|_{V} \leq \varepsilon(N), \text { where } \varepsilon(N) \rightarrow \infty \text {. }
$$

Let $P: H \rightarrow H^{N}$ be the canonical orthogonal projection onto $H^{N}$. Then, from the approximation property (APP), it is a trivial matter to see that $P^{N} z \rightarrow z$ as $N \rightarrow \infty$, for $z \in$ $H$. For the finite demensional regulator problem $(L Q R)_{\alpha}^{N}$ we choose

$$
B^{N}=P^{N} B \quad \text { and } \quad C^{N}=C P^{N} .
$$


Then the conditions $(A 2)(\mathrm{i})$,(ii) follow from Remark 4.2 .

To obtain a representation $A_{\epsilon}^{N}$ of $A_{\varepsilon}$ on $H^{N}$, consider the restriction of the sesquilinear form $a_{\epsilon}(\cdot, \cdot)$ to $H^{N} \times H^{N}$. We then have a representation $A_{\epsilon}^{N}$ of $A_{\epsilon}$ satisfying

$$
a_{\epsilon}(z, w)=<-A_{\epsilon}^{N} z, w>\quad \text { and } \quad a_{\epsilon}(z, w)=<-\left(A_{\epsilon}^{N}\right)^{*} w, z>
$$

for all $z, w \in H^{N}$. Equation (4.15) follows from the fact that $H^{N}$ is a real Hilbert space. We know also that $A_{\epsilon}^{N}=\left(A_{\epsilon}^{N}\right)^{*}$, since $A_{\epsilon}=A_{\epsilon}^{*}$.

Remark 4.4. Since $H^{N} \subset H$, by equation (4.15), it is easy to see that for any $\lambda \in \sigma\left(A_{\epsilon}^{N}\right)$, $\mathcal{R} e \lambda \leq-\gamma \leq-\epsilon$.

Let $S^{N}(t)$ be the $C_{0}$-semigroup generated by $A_{\epsilon}^{N}$. Then the conditions $(A 1)(\mathrm{i})$,(ii) follow from the results of Banks and Kunish [4, Lemma 3.2]. Note that $S^{N}(t)=\left(S^{N}(t)\right)^{*}$. For the condition $(A 3)(\mathrm{i})$ we need a certain preservation of exponential stabilizability under approximation ( (POES) in [4]). The following result is taken from [4, Lemma 3.3].

Theorem 4.5. Let $\left(A_{\epsilon}, B\right)$ be (exponentially) stabilizable. Suppose that the approximation condition (APP) holds. Then the approximations defined through equations (4.14)-(4.15) satisfy the condition $(A 3)(\mathrm{i})$, i.e., the family of pairs $\left(A_{\epsilon}^{N}, B^{N}\right)$ is uniformly stabilizable.

By the dual arguments of Theorem 4.5 we can see that the condition (A3) (ii) holds under the assumption that $\left(A_{\varepsilon}, C\right)$ is detectable. We summarize our discussion up to this point as the following theorem.

Theorem 4.6. Let $\left(A_{\epsilon}+\alpha I, B\right)$ be stabilizable and $\left(A_{\epsilon}+\alpha I, C\right)$ be detectable. Let $A_{\epsilon}^{N}$, $B^{N}, C^{N}$ be defined as in equations (4.14) and (4.15). Then we have

$$
\Pi_{\alpha, \epsilon}^{N} P^{N} z \longrightarrow \Pi_{\alpha, \epsilon} z, \quad z \in H
$$

and

$$
S^{N}(t) P^{N} z \longrightarrow S(t) z, \quad z \in H
$$

where the convergence is uniform in $t$ on bounded subsets of $[0, \infty), P^{N}$ is the orthogonal projection onto $H^{N}$, and $\Pi_{\alpha, c}^{N}$ satisfies

$$
\left(A_{\epsilon}^{N}+\alpha I^{N}\right)^{*} \Pi_{\alpha, \epsilon}^{N}+\Pi_{\alpha, \epsilon}^{N}\left(A_{\epsilon}^{N}+\alpha I^{N}\right)-\Pi_{\alpha, \epsilon}^{N} B^{N}\left(B^{N}\right)^{*} \Pi_{\alpha, \epsilon}^{N}+\left(C^{N}\right)^{*} C^{N}=0 .
$$


Remark 4.7. Note that $S(t)=\hat{S}(t) e^{-\alpha t}, S^{N}(t)=\hat{S}^{N}(t) e^{-\alpha t}$, where $S(t), S^{N}(t), \hat{S}(t)$ and $\hat{S}^{N}(t)$ are semigroups generated by $A_{\epsilon}, A_{\epsilon}^{N}, A_{\epsilon}+\alpha I$ and $A_{\epsilon}^{N}+\alpha I^{N}$, respectively.

Next, consider the matrix representations of operators on the space $H^{N}$. Let the approximate solution $z^{N}(t, x)$ of $z(t, x)$ on $H^{N}$ be given by

$$
z^{N}(t, x)=\sum_{i=1}^{N} z_{i}^{N}(t) h_{i}^{N}(x)
$$

for some $z_{i}^{N}(t) \in \mathbf{R}, i=1, \cdots, N$. Then, from equations (4.14) and (4.15), we have a finite dimensional ODE system

$$
\left[G^{N}\right] \frac{d}{d t}\left\{z^{N}(t)\right\}=\left[\tilde{A}_{\epsilon}^{N}\right]\left\{z^{N}(t)\right\}+\left\{\tilde{B}^{N}\right\} u(t)
$$

where $\left\{z^{N}(t)\right\}=\left[z_{1}^{N}(t), \cdots, z_{N}^{N}(t)\right]^{T}$,

$$
\begin{aligned}
& {\left[G^{N}\right]=\left[<h_{j}^{N}, h_{i}^{N}>\right]_{N \times N}} \\
& =\frac{1}{6(N+1)}\left[\begin{array}{ccccccc}
4 & 1 & 0 & 0 & & \ldots & 0 \\
1 & 4 & 1 & 0 & & \ldots & 0 \\
0 & 1 & 4 & 1 & & \ldots & 0 \\
& & \ddots & \ddots & \ddots & & \\
\vdots & & \ddots & \ddots & \ddots & \ddots & \vdots \\
0 & & & & 1 & 4 & 1 \\
0 & & \cdots & & 0 & 1 & 4
\end{array}\right]_{N \times N} \\
& {\left[\tilde{A}_{\epsilon}^{N}\right]=\epsilon(N+1)\left[\begin{array}{ccccccc}
-2 & 1 & 0 & 0 & & \ldots & 0 \\
1 & -2 & 1 & 0 & & \ldots & 0 \\
0 & 1 & -2 & 1 & & \ldots & 0 \\
& & \ddots & \ddots & \ddots & & \\
\vdots & & \ddots & \ddots & \ddots & \ddots & \vdots \\
0 & & & & 1 & -2 & 1 \\
0 & & \ldots & & 0 & 1 & -2
\end{array}\right]_{N \times N}} \\
& \left\{\tilde{B}^{N}\right\}=\left[<b, h_{1}^{N}>,<b, h_{2}^{N}>, \cdots,<b, h_{N}^{N}>\right]^{T} \text {, }
\end{aligned}
$$

where $\left\langle b, h_{j}^{N}\right\rangle=\int_{0}^{1} b(x) h_{j}^{N}(x) d x, 1 \leq j \leq N$. Since $\left[G^{N}\right]$ is invertible, by multiplying $\left[G^{N}\right]^{-1}$ to both sides of $(4.20)$, we get

$$
\frac{d}{d t}\left\{z^{N}(t)\right\}=\left[A_{c}^{N}\right]\left\{z^{N}(t)\right\}+\left\{B^{N}\right\} u(t), \quad\left\{z^{N}(0)\right\}=\left\{z_{0}^{N}\right\}
$$

where

$$
\left[A_{\epsilon}^{N}\right]=\left[G^{N}\right]^{-1}\left[\tilde{A}_{\epsilon}^{N}\right], \quad\left\{B^{N}\right\}=\left[G^{N}\right]^{-1}\left\{\tilde{B}^{N}\right\}
$$


and $\left\{z_{0}^{N}\right\}=\left[G^{N}\right]^{-1}\left[<z_{0}, h_{1}^{N}>, \cdots,<z_{0}, h_{N}^{N}>\right]^{T}$.

Next, consider a representation $C^{N}$ of the operator $C$ on $H^{N}$. It is easy to see that $C^{N}: H^{N} \rightarrow \mathbf{R}^{k}$ is given by

$$
\left[C^{N}\right]=\left[\tilde{h}_{j}^{N}\left(\bar{x}_{i}\right)\right]_{k \times N}
$$

where $\tilde{h}_{j}^{N}\left(\bar{x}_{i}\right)=\frac{1}{2 \delta} \int_{\bar{x}_{i}-\delta}^{\bar{x}_{i}+\delta} h_{j}^{N}(x) d x, 1 \leq i \leq k, 1 \leq j \leq N$.

Finally, we have a finite dimensional Riccati equation (4.18) and the corresponding feedback gain operator $K_{\alpha, \epsilon}^{N}$ given by

$$
K_{\alpha, \epsilon}^{N}=-\left(B^{N}\right)^{*} \Pi_{\alpha, \epsilon}^{N}
$$

Therfore, the closed loop system (4.24) can be represented by

$$
\frac{d}{d t}\left\{z^{N}(t)\right\}=\left(A_{\epsilon}^{N}+B^{N} K_{\alpha, \epsilon}^{N}\right)\left\{z^{N}(t)\right\}, \quad\left\{z^{N}(0)\right\}=\left\{z_{0}^{N}\right\}
$$

Now, we discuss an algorithm for finding the unique nonnegative self-adjoint Riccati solution for equation (4.18). We employ the Potter's method [30] to obtain $\Pi_{\alpha, \epsilon}^{N}$. The first step in Potter's method is to form $2 N \times 2 N$ matrix

$$
M^{N}=\left[\begin{array}{cc}
\left(A_{\epsilon}^{N}+\alpha I^{N}\right)^{*} & \left(C^{N}\right)^{*} C^{N} \\
B^{N}\left(B^{N}\right)^{*} & -\left(A_{\epsilon}^{N}+\alpha I^{N}\right)
\end{array}\right] .
$$

Next, find all eigenvalues and eigenvectors of $M^{N}$ and form the matrix

$$
Z^{N}=\left[\begin{array}{c}
Q_{1}^{N} \\
Q_{2}^{N}
\end{array}\right]
$$

where the columns of $Z^{N}$ are the eigenvectors of $M^{N}$ corresponding to the eigenvalues with positive real part. When eigenvalues occur in complex conjugate pairs, so do the eigenvectors. In this case, the real and imaginary part of the eigenvector each forms a column of $Z^{N}$. Finally, the solution to the Riccati equation (4.18) is given by the formula $\Pi_{\alpha, \epsilon}^{N}=Q_{1}^{N}\left(Q_{2}^{N}\right)^{-1}$.

Remark 4.8. From the numerical results we found that the Riccati solution operators $\Pi_{\alpha, \varepsilon}^{N}$ blow up when the viscosity $\epsilon>0$ goes to 0 for fixed $\alpha>0$. Also, when $\alpha$ goes to infinity with $\epsilon$ fixed the same phenomenon has been observed. 
Finally, the finite dimensional approximation for the controlled nonlinear Burgers' equation (3.33) is given by

$$
\frac{d}{d t}\left\{z^{N}(t)\right\}=\left(\left[A_{\varepsilon}^{N}\right]+\left\{B^{N}\right\}\left[K_{\alpha, \epsilon}^{N}\right]\right)\left\{z^{N}(t)\right\}+f^{N}\left(\left\{z^{N}(t)\right\}\right), \quad\left\{z^{N}(0)\right\}=\left\{z_{0}^{N}\right\}
$$

where $\left[A_{\epsilon}^{N}\right],\left\{B^{N}\right\}$ are defined as in equation (4.25), and

$$
\begin{aligned}
& f^{N}\left(\left\{z^{N}(t)\right\}\right)=\left[G^{N}\right]^{-1} \tilde{f}^{N}\left(\left\{z^{N}(t)\right\}\right), \\
& \tilde{f}^{N}\left(\left\{z^{N}(t)\right\}\right)=\frac{1}{6}\left[\begin{array}{c}
z_{1}^{N}(t) z_{2}^{N}(t)+\left(z_{2}^{N}(t)\right)^{2} \\
-\left(z_{1}^{N}(t)\right)^{2}-\left(z_{1}^{N}(t)\right)\left(z_{2}^{N}(t)\right)+\left(z_{2}^{N}(t)\right)\left(z_{3}^{N}(t)\right)+\left(z_{3}^{N}(t)\right)^{2} \\
\vdots \\
-\left(z_{N-2}^{N}(t)\right)^{2}-\left(z_{N-2}^{N}(t)\right)\left(z_{N-1}^{N}(t)\right)+\left(z_{N-1}^{N}(t)\right)\left(z_{N}^{N}(t)\right)+\left(z_{N}^{N}(t)\right)^{2} \\
-\left(z_{N-1}^{N}(t)\right)^{2}-\left(z_{N-1}^{N}(t)\right)\left(z_{N}^{N}(t)\right)
\end{array}\right],
\end{aligned}
$$

where $\left[G^{N}\right]$ is defined by equation (4.21). To solve the nonlinear ODE system (4.31) we use the 4-th order Runge-Kutta method [33].

Throughout the rest of this section, we discuss how our results work for relaxation of "steep" gradient of the solution for Burgers' equation through numerical experiments.

For numerical examples, the length $\ell$ for space domain, the Reynolds nummber, Re, the initial function $z_{0}(\cdot) \in H_{0}^{1}(0,1)$ and the control input function $b(\cdot) \in L^{2}(0,1)$ will be chosen as $1,60, \sin \pi x$ and $e^{x}$, respectively. Thus the governing equation is given by

$$
\begin{aligned}
\frac{\partial}{\partial t} z(t, x) & =\frac{1}{60} \frac{\partial^{2}}{\partial x^{2}} z(t, x)-z(t, x) \frac{\partial}{\partial x} z(t, x)+e^{x} \int_{0}^{1} k_{\alpha, \epsilon}(s) z(t, s) d s \\
z(t, 0) & =z(t, 1)=0 \\
z(0, x) & =\sin \pi x,
\end{aligned}
$$

where the feedback gain function $k_{\alpha, \epsilon}(\cdot) \in L^{2}(0,1)$ will be determined by the desired degree $\alpha>0$ of stability and the action of output operator $C$.

The "robustness" of the feedback controller exhibited, for example, in Figure 2, will be discussed in Example 3. For this particular example, Reynolds numbers 60, 80, 100 and 120 are chosen.

Remark 4.9. (i) From the numerical experiments, we found that if Reynolds number, Re, is less than 60 , then the diffusion phenomena dominate convection phenomena. In this 
case, the formation of steep gradient due to convection term $-z(t, x) \frac{\partial}{\partial x} z(t, x)$ of the openloop solution, i.e., $k_{\alpha, c}(\cdot) \equiv 0$ in equation (4.32), is not clear. But, for Reynolds number greater than 60 , the open-loop solution creates "sharp" gradient in finite time, (see Figures $1,10,12$ and 14). Of course, the solution dies out eventually, because of the diffusion term $\frac{1}{\operatorname{Re}} \frac{\partial^{2}}{\partial x^{2}} z(t, x)$.

(ii) The control input function $b(x)=e^{x}$ is defined for all $x \in[0,1]$. Thus, the feedback control acts on the whole domain $[0,1]$. But, one can choose any $L^{2}$-function $b(\cdot) \in L^{2}(0,1)$ satisfying the stabilizability condition in Lemma 3.7. In fact, $b(x)=e^{x}$ satisfies the stabilizability condition for any desired degree of stability $\alpha>0$, since the coefficients $b_{n}, n=1,2, \cdots$, representing input function $b(\cdot)$ are not zero, i.e., $b_{n}=<$ $b(\cdot), \sin \pi x>_{L^{2}(0,1)}=\int_{0}^{1} e^{x} \sin \pi x d x \neq 0$ for all $n=1,2, \cdots$, (see Lemma 3.7).

(iii) The initial function $z_{0}(x)=\sin \pi x$ is chosen for our numerical experiments. Other typical $H_{0}^{1}$-functions such as the "hat function" defined by

$$
z_{0}(x)= \begin{cases}2 x, & x \in\left[0, \frac{1}{2}\right] \\ -2 x+2, & x \in\left[\frac{1}{2}, 1\right]\end{cases}
$$

can be used for initial data. But, we found that the solution of Burgers' equation (4.32) with initial data $z_{0}(x)$ replaced by the hat function has almost similar phenomena, such as the creation or relaxation of steep gradients, as those of sloution with initial data $z_{0}(x)=\sin \pi x$.

To show trajectories of open-loop and closed-loop solutions, the order $N$ of approximation is chosen as $N=32$ for both cases. And the corresponding trajectories from time $t=0.0$ to $t=1.0$ will be shown. The convergence of the feedback gain functions $k_{\alpha, \epsilon}(\cdot) \in L^{2}(0,1)$ will be shown for $N=8,16,32,64$ and 128 .

Example 1. (Bounded Input/Output)

The observation operator $C \in \mathcal{L}\left(L^{2}(0,1), \mathbf{R}^{3}\right)$ for this example is given by

$$
C(z)=(\tilde{z}(0.3), \tilde{z}(0.5), \tilde{z}(0.75))
$$

where $\tilde{z}(\bar{x})$ is the average value of $z(\cdot) \in L^{2}(0,1)$ in a small neighborhood of $\bar{x}, \bar{x}=$ $0.3,0.5,0.75$, and defined by equation $(3.11), \tilde{z}(\bar{x})=\frac{1}{2 \delta} \int_{\bar{x}-\delta}^{\bar{x}+\delta} z(s) d s$. Here, $\delta>0$ is chosen so small that each open interval $(\bar{x}-\delta, \bar{x}+\delta)$ is contained in the whole domain $(0,1)$. The desired degree $\alpha$ of stability are chosen 0.3 and 0.6 for Figures 3 and 5, respectively. For 
both cases, $n_{\alpha}=\max \left\{n \in \mathbf{N}: \alpha-\frac{1}{60} n^{2} \pi^{2} \geq 0\right\}=1$ and hence the set $X_{\alpha, 1}$ defined in Lemma 3.7 is empty. Thus, all assumptions in Theorem 3.9 are satisfied.

The feedback gain functions $k_{\alpha, \varepsilon}(\cdot)$ are given in Figures 2 and 4 . From these plots, it is easy to see that control action is concentrated on the location of sensors. This phenomenon is natural, since the optimal control is obtained to minimize the cost functional $J$ defined by equation (3.16) whose first term $\|\hat{y}(t)\|_{Y}^{2}=\|C \hat{z}(t)\|_{\mathbf{R}^{3}}^{2}=\sum_{i=1}^{3}\left|\tilde{\hat{z}}\left(\bar{x}_{i}\right)\right|^{2}$, where $\bar{x}_{i}=0.3,0.5$ and 0.75 for $i=1,2$ and 3 , respectively. The corresponding closed-loop trajectories are shown in Figure 3 (for $\alpha=0.3$ ) and Figure 5 (for $\alpha=0.6$ ). From Figures 3 and 5 , we can see how the controllers contribute to stabilization of the steep gradient as well as the solution itself.

\section{Example 2. (Identity Output Operator)}

For this example, we take the identity operator $I$ on $L^{2}(0,1)$ for the output operator $C$. In this case, the ouput space $Y$ is $L^{2}(0,1)$. The convergence of gain functions and corresponding closed-loop trajectories for $\alpha=0.3$ and 0.6 are shown in Figures 6-7 and 8-9, respectively. Since the observation operator is identity, this example gives the information about the maximal control action. We note the following observation concerning the convergence rate of gain function. Theoretically, the rate is $O\left(\frac{1}{N}\right)[19, \mathrm{p} .15]$. But, in this example, the rate seems to be faster than $O\left(\frac{1}{N}\right)$, (see Figures 2 and 4 ). Another observation is concerned with the location of maximal control action. The location moves to the left portion of domain as the degree of stability $\alpha>0$ increases. In other words, we should put more action on the front part of domain to get a higher exponential decay rate $\alpha>0$. (See also Figures 2 and $4)$.

Example 3. (Robustness)

In this example we show the robustness of the feedback controller showed in Figure 2. The feedback controller is obtained from the control system with $\operatorname{Re}=60, \alpha=0.3, b(x)=e^{x}$ and the ouput operator $C$ defined by equation (4.34).

Figures 2, 10, 12 and 14 show open-loop trajectories for Reynolds numbers 60, 80, 100 and 120, respectively. The corresponding closed-loop trajectories are shown in Figures $2,11,13$ and 15 , respectively. The order $N$ of approximation is chosen as $N=32$ for $\operatorname{Re}=60,80,100$ and $N=64$ for $\operatorname{Re}=120$. From these examples, it is easy to see that the feedback controller obtained for $\mathrm{Re}=60$ stabilizes the steep gradient of the solution for 
Burgers' equation with various Reynolds numbers. However, we see that the sharp gradient is relaxed slowly as Reynolds number increases, (see Figures 3,11,13 and 15). Note that, from Theorem 3.10, the closed-loop solution $z(t)$ of the nonlinear system (3.33) satisfies the stability property

$$
\|z(t)\|_{H_{0}^{1}} \leq 2 M e^{-\alpha t}\left\|z_{0}(\cdot)\right\|_{H_{0}^{1}} \cdot
$$

Although the exponential decay rate $\alpha$ is independent of Reynolds number, the constant $M=M(\alpha, \operatorname{Re})$ depends strongly on the Reynolds number, Re.

\section{Conclusion}

In this paper, we considered a feedback control problem for a nonlinear equation, in particular, Burgers' equation. The method consists of linearization of the nonlinear equation. We used the linear quadratic regulator (LQR) problem to find optimal feedback gains. The linearized equation is the heat equation. It was also proved that, under appropriate selection of the input functions and "weights location", the LQR problem for the linearized problem is detectable and stabilizable. We then analysed a "shifted quadratic cost" to construct gains which produce a fixed decay rate. In particular, we showed that the closed-loop system satisfies the inequality

$$
\left\|z\left(t, 0 ; z_{0}\right)\right\|_{H_{0}^{1}} \leq M(\epsilon) e^{-\alpha t}\left\|z_{0}\right\|_{H_{0}^{1}}
$$

where $\alpha>0$ does not depend on the Reynolds number, but $M(\epsilon)$ does, (see Theorem 3.10).

We also developed a numerical scheme for computing the feedback functional gains. Several numerical experiments were performed and the following observations were made:

1) The functional gains depend strongly on the "weight location". For example, if the output operator $C$ is given by

$$
C(z)=(\tilde{z}(.3), \tilde{z}(.5), \tilde{z}(.75)),
$$

then the gain function is concentrated on the weight location, $0.3,0.5$ and 0.75 , (Figures 2 and 4).

2) The closed-loop nonlinear system is stabilized (as predicted) by linear feedback laws. Moreover, the steep gradients (for $\epsilon \approx 0$ ) are smoothed out by feedback.

3) To test the "robustness" of the feedback control law, one experiment was performed. We obtained the functional gain $k_{\alpha, \epsilon}^{1}(\cdot)$ from the control system at the Reynolds number, 
$\operatorname{Re}=60$, and applied it to the closed-loop system

$$
\frac{\partial}{\partial t} z(t, x)=\frac{1}{\operatorname{Re}} \frac{\partial^{2}}{\partial x^{2}} z(t, x)-z(t, x) \frac{\partial}{\partial x} z(t, x)+e^{x} \int_{0}^{1} k_{\alpha, \epsilon}^{1}(s) z(t, s) d s
$$

at $\operatorname{Re}=80,100$ and 120. The closed-loop responses are shown in Figures 11, 13 and 15. Although the performance was decreased, the system (5.1) was still stabilized and smoothed out. These results provided some insight into the possibility of using linear feedback laws for nonlinear distributed parameter systems. 


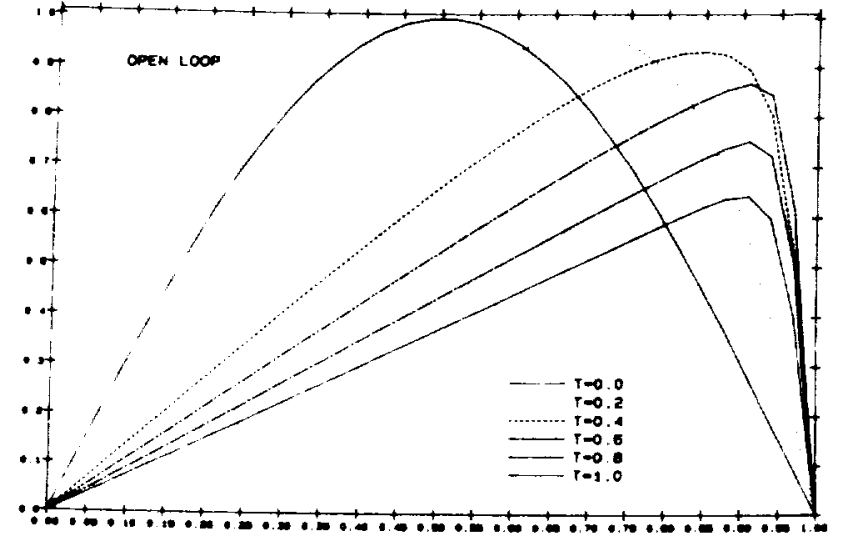

Firure I Open Loop $(N=32, \operatorname{Re}=60,20(x)=$ in $\pi x)$

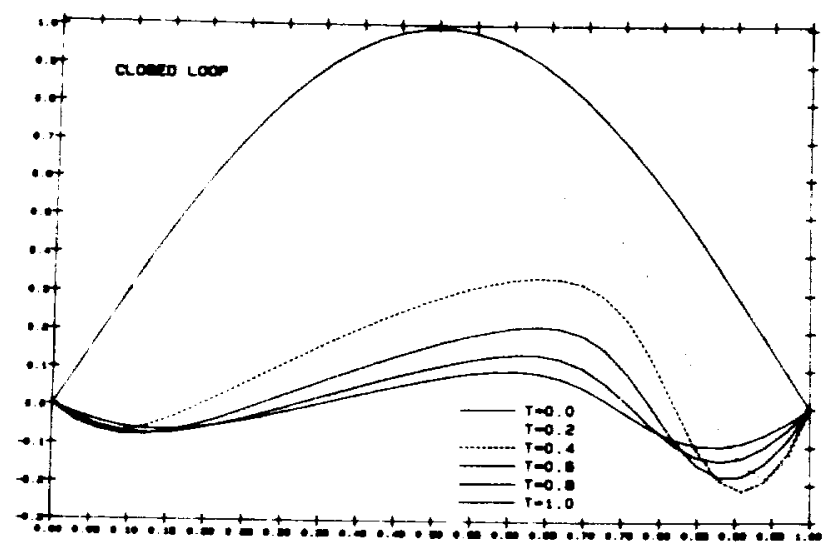

Figure 3 Clowed loop (foedbect by $k_{a, d}(1)$

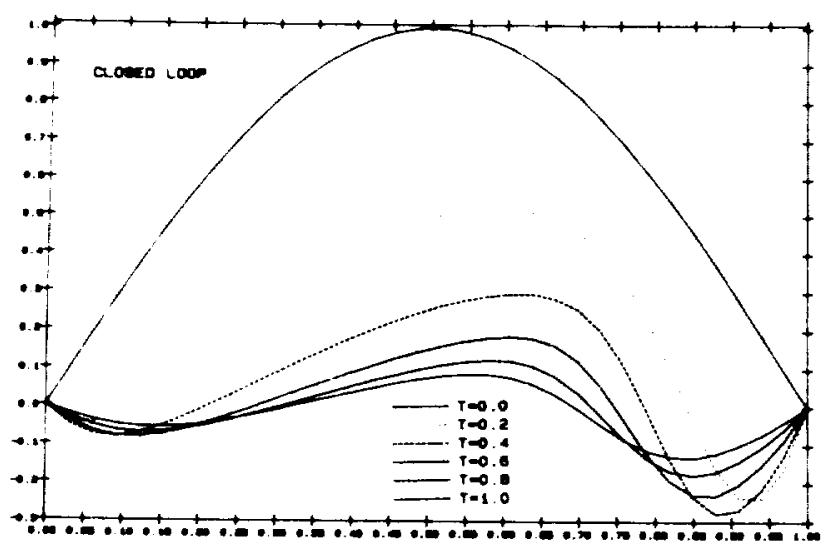

Figure 5. Clooed Loop (feedback by $k_{2}^{2}$ (f) )

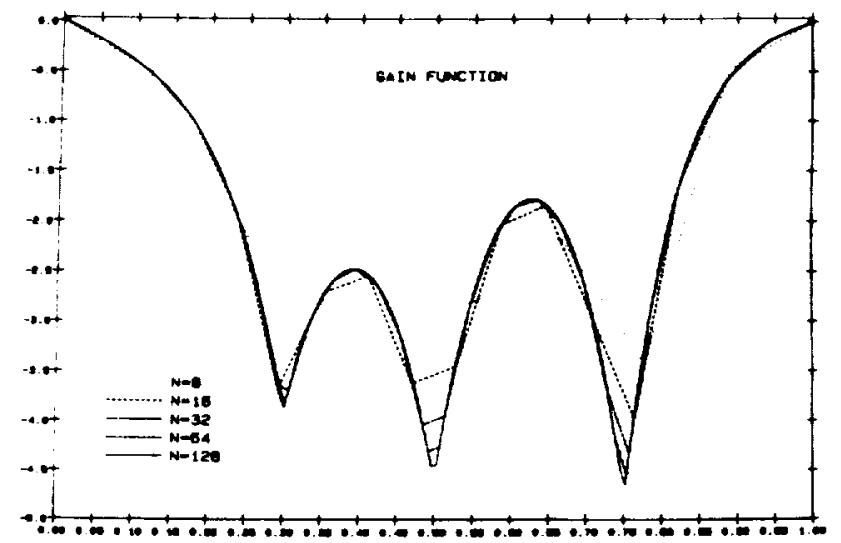

Figure 2. Gain Punction $h_{a d}^{1}(\cdot)(a=0.3, C(z)=(\tilde{y}(0.3), z(0.5), \dot{z}(0.75)))$

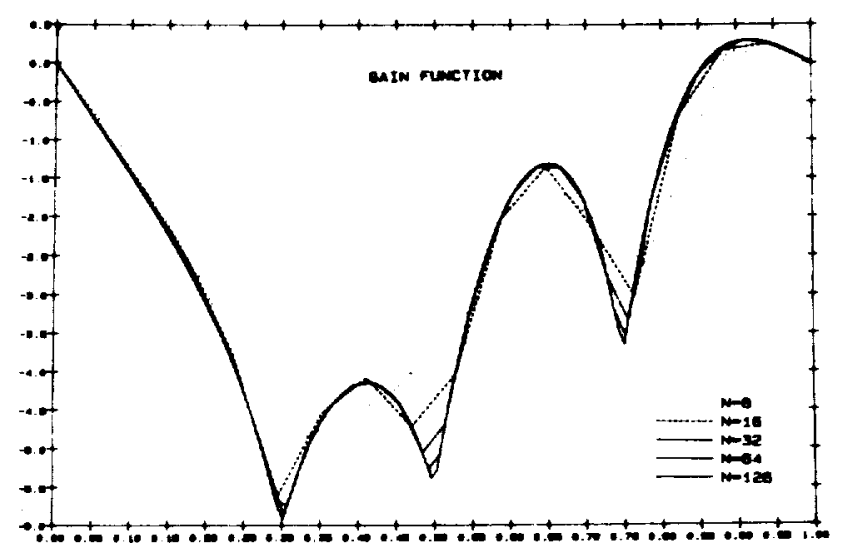

Figure 4. Gain Function $t_{\alpha, d}^{2}()(a=0.8, C(x)=(i(0.3), y(0.6), z(0.78)))$

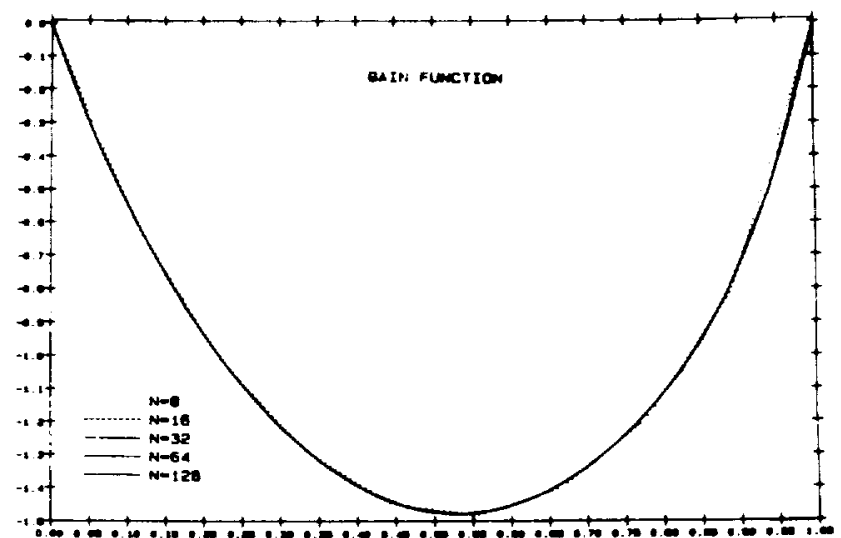

Figure 6. Gevin Function ta, ()$(\alpha=0.3, C=I)$ 


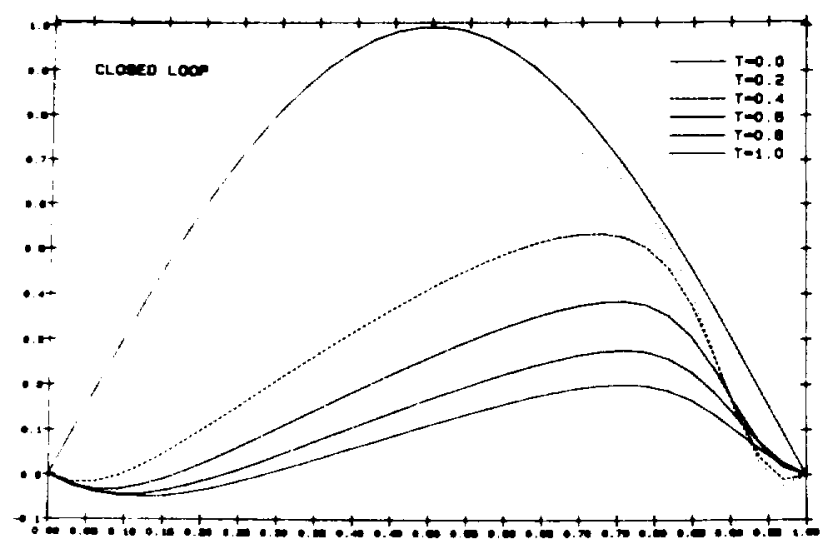

Figure 1 Clowed Loop (feedback by $\left.k_{a, e}^{3}()\right)$

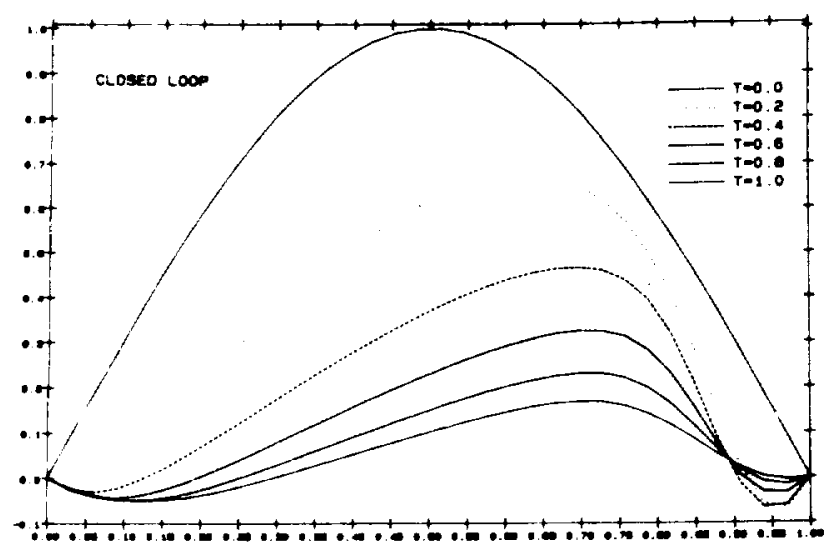

Figure 9 Clowed Loop (feedback by $k_{a x}($ ))

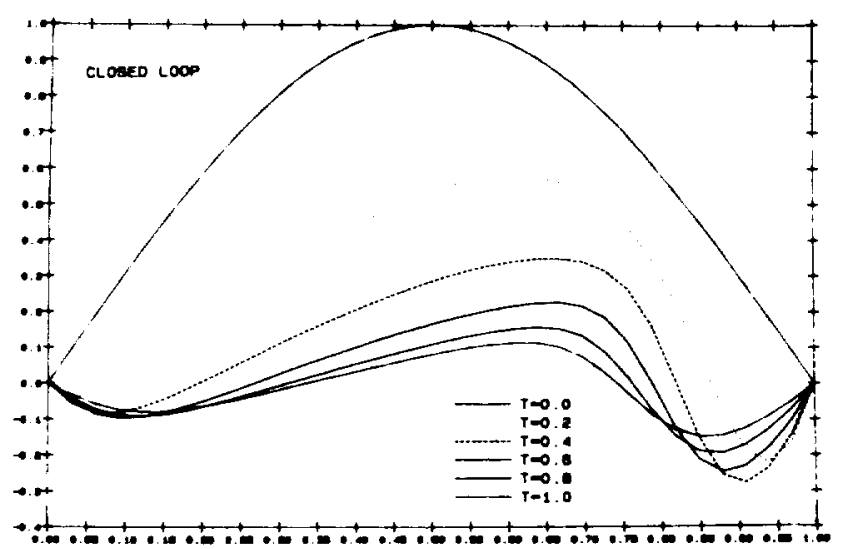

Figure 11. Cloned Loop (Re $=00$, feedbect by $k_{a, 4}^{1}($ )

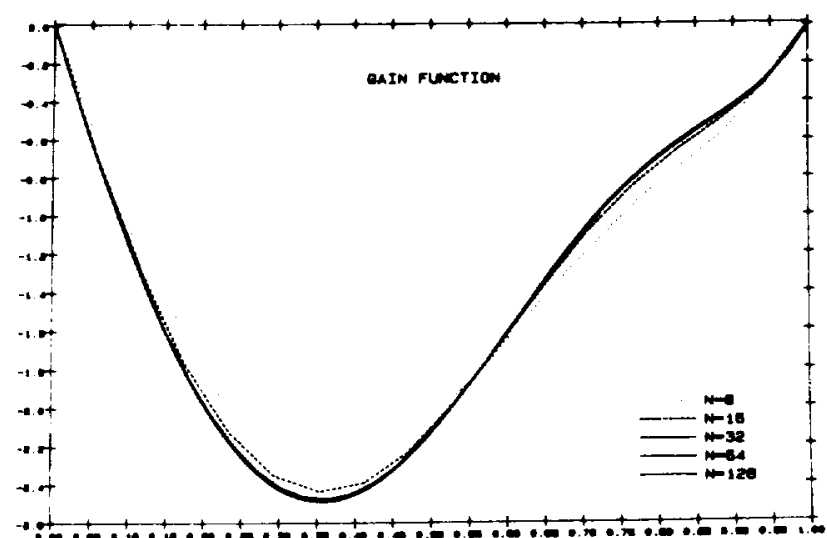

Figure 8. Gain Function $b_{a, d}^{4}()(a=0.6, C=n)$

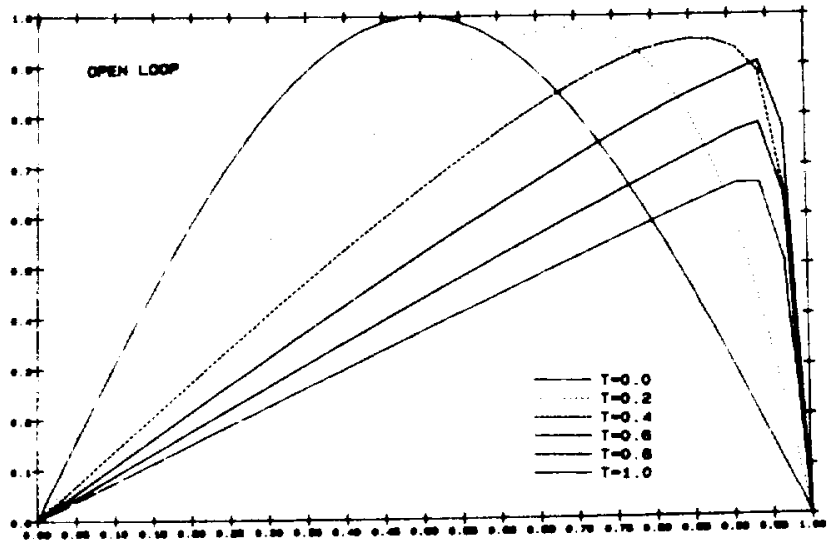

Figure 10. Open Lmp $\left(N=32\right.$, Re $\left.=80, x_{0}(x)=\sin \pi x\right)$

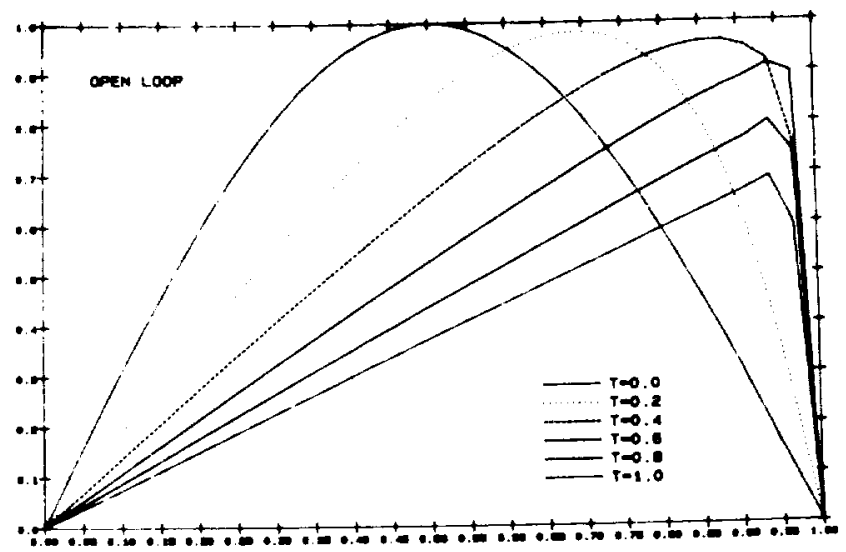

Figure 12. Open Loop $(N=32, R e=100,20(x)=\sin \pi x)$ 


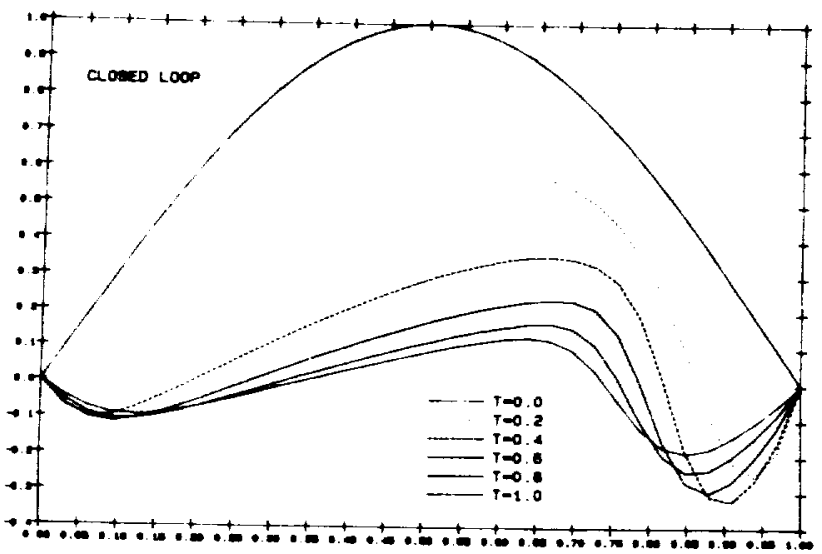

Figure 13. Cloned Loop (Re $=100$, feedbuck by $\left.k_{a, 4}^{1}()\right)$

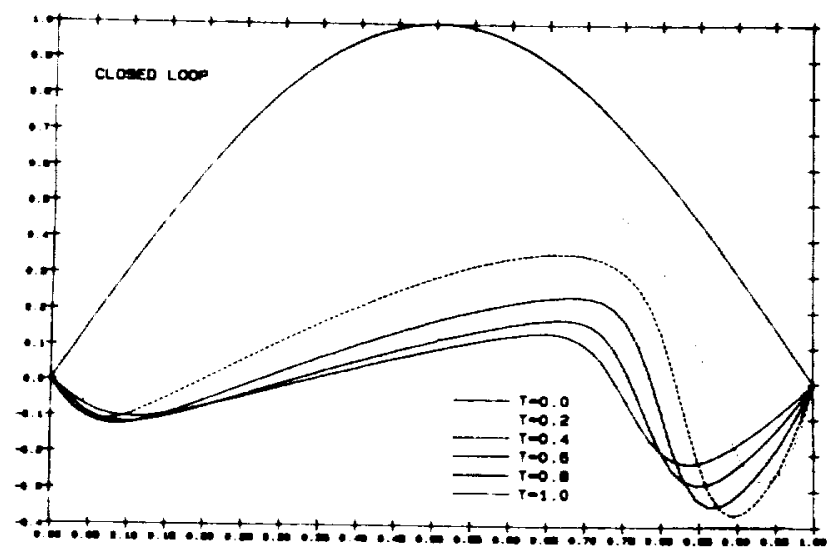

Figure 15 Cloned Loop $\left(N=64, R_{r}=120\right.$, feed bect by $k_{\alpha}^{1}(1)$

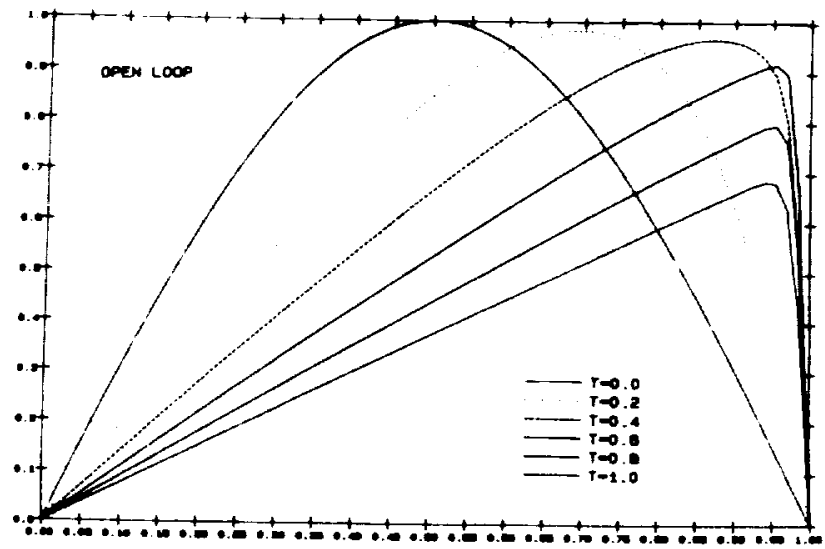

Figure 14. Open Lrop $\left(N=64,8 p-120, z_{0}(x)=\operatorname{man}=x\right)$ 


\section{References}

[1]. Anderson, B. D. O. and Moore, J. B., "Linear system optimisation with prescribed degree of stability", Proc. IEEE, Vol. 116, No. 12, 1969, 2083-2087.

[2]. Banks, H. T. and Burns, J. A., "Hereditary control problems: numerical methods based on averaging approximation", SIAM J. Control and Optim., Vol. 16, No. 2, 1978, 169-208.

[3]. Banks, H. T., Burns, J. A. and Cliff, E. M., "Parameter estimation and identification for systems with delays", SIAM J. Control and Optim., Vol. 19, No. 6, 1981, 791-828.

[4]. Banks, H. T. and Kunish, K., "The linear regulator problem for parabolic systems", SIAM J. Control and Optim., Vol. 22, No. 5, 1984, 684-698.

[5]. Burgers, J. M., "Mathematical examples illustrating relations occuring in the theory of turbulent fluid motion", Trans. Roy. Neth. Acad. Sci., Amsterdam, Vol. 17, 1939, 1-53.

[6]. Burgers, J. M., "A mathematical model illustrating the theory of turbulence", Adv. in Appl. Mech., Vol. 1, 1948, 171-199.

[7]. Burgers, J. M., "Statistical problems connected with asymptotic solution of onedimensional nonlinear diffusion equation", in M. Rosenblatt and C. van Atta (eds.), Statistical Models and Turbulence, Springer, Berlin, 1972, 41-60.

[8]. Chen, G., Wang, H. K. and Weerakoon, S., “An initial value control problem for Burgers' equation", in F. Kappel, K. Kunish and W. Schappacher (eds.), Distributed Parameter Systems, Lecture Notes in Control and Information Sciences, Vol. 75, 1985, 52-76.

[9]. Cole, J. D., "On a quasi-linear parabolic equation occuring in aerodynamics", Quart. Appl. Math., Vol. IX, No. 3, 1951, 225-236.

[10]. Conway, J. B., A Course in Functional Analysis, Springer-Verlag, New York, 1985.

[11]. Curtain, R. F., "Stability of semilinear evolution equations in Hilbert space", J. Math. Pures et Appl, Vol. 63, 1984, 121-128.

[12]. Fletcher, C. A. J., "Burgers' equation : a model for all reasons", in J. Noye (ed.), Numerical Solution of Partial Differential Equations, North-Holland, 1982, 139-225.

[13]. Gibson, J. S., “The Riccati integral equations for optimal control problems on Hilbert spaces", SIAM J. Control and Optim., Vol. 17, 1979, 537-565. 
[14]. Gibson, J. S., "Linear-quadratic optimal control of hereditary differential systems: infinite dimensional Riccati equations and numerical approximations", SIAM J. Control and Optim., Vol. 21, No. 1, 1983, 95-139.

[15]. Gibson, J. S. and Rosen, I. G., "Shifting the closed-loop spectrum in the optimal linear quadratic regulator problem for hereditary systems", Institute for Computer Applications for Science and Engineering, ICASE Report 86-16, 1986, NASA Langley Research Center, Hampton, VA.

[16]. Glimm, J. and Lax, P., Decay of Solutions of Systems of Nonlinear Hyperbolic Conservation Laws, Amer. Math. Soc. Memoir, No. 101, A.M.S., Providence, 1970.

[17]. Henry, D., Geometric Theory of Semilinear Parabolic Equations, Springer-Verlag, New York, 1981.

[18]. Hopf, E., "The partial differential equation $u_{t}+u u_{x}=\mu u_{x x}$ ", Comm. Pure and Appl. Math, Vol. 3, 1950, 201-230.

[19]. Ito, K., "Strong convergence and convergence rates of approximating solutions for algebraic Riccati equations in Hilbert spaces", LCDS/CCS Report \#87-15, Brown University, 1987.

[20]. Kielhöfer, H., "Stability and semilinear evolution equations in Hilbert space", Arch. Rat. Mech. Anal., Vol. 57, 1974, 150-165.

[21]. Lasiecka, I., "Unified theory for abstract parabolic boundary problems - a semigroup approach", Appl. Math. Optim, Vol. 6, 1980, 287-333.

[22]. Lax, P. D., Hyperbolic Systems of Conservation Laws and the Mathematical Theory of Schock Waves, CBMS-NSF Regional Conference Series in Applied Mathematics, Vol. 11, SIAM, 1973.

[23]. Lighthill, M. J., "Viscosity effects in sound waves of finite amplitude", in G. K. Bachelor and R. M. Davies (eds.), Surveys in Mechanics, Cambridge University Press, Cambridge, $1956,250-351$.

[24]. Maslov, V. P., "On a new principle of superposition for optimization problems", Uspekhi Mat. Nauk, Vol. 42, No. 3, 1987, 39-48.

[25]. Maslov, V. P., "A new approach to generalized solutions of nonlinear systems", Soviet Math. Dokl, Vol. 35, No. 1, 1987, 29-33. 
[26]. Oleinik, O. A., "Discontinuous solutions of nonlinear differential equations." Uspsekhi Math. Nauk, Vol. 12, 1957, 3-73, (English Translation Amer. Math. Soc. Trans. , Series 2, 26, 95-172).

[27]. Pazy, A., Semigroups of Linear Operators and Applications to Partial Differential Equations, Springer-Verlag, New York, 1983.

[28]. Pritchard, A. J. and Salamom, D., "The linear quadratic control problem for infinite dimensional systems with unbounded input and output operators", SIAM J. Control and Optimization, Vol. 25, No. 1, 1987, 121-144.

[29]. Rankin, S. M., "Semilinear evolution equations in Banach spaces with application to parabolic partial differential equations", Preprint, 1989.

[30]. Russell, D. L., Mathematics of Finite-Dimensional Control Systems: Theory and Design, Marcel Dekker Inc., 1979.

[31]. Schultz, M. H., Spline Analysis, Prentice-Hall, N.J., 1973.

[32]. Smoller, J., Shock Waves and Reaction-Diffusion Equations, Springer-Verlag, 1983.

[33]. Stoer, J. and Bulirsch, R., Introduction to Numerical Analysis, Springer-Verlag, New York, 1980

[34]. Walker, J. A., Dynamical Systems and Evolution Equations, Theory and Applications, Plenum Press, New York, 1980.

[35]. Weerakoon, S., An Initial Value Control Problem for the Burgers' Equation, Ph.D. Thesis, Department of Mathematics, Pennsylvania State University, December, 1984.

[36]. Wloka, J., Partial Differential Equations, Cambridge University Press, Cambridge, 1987. 



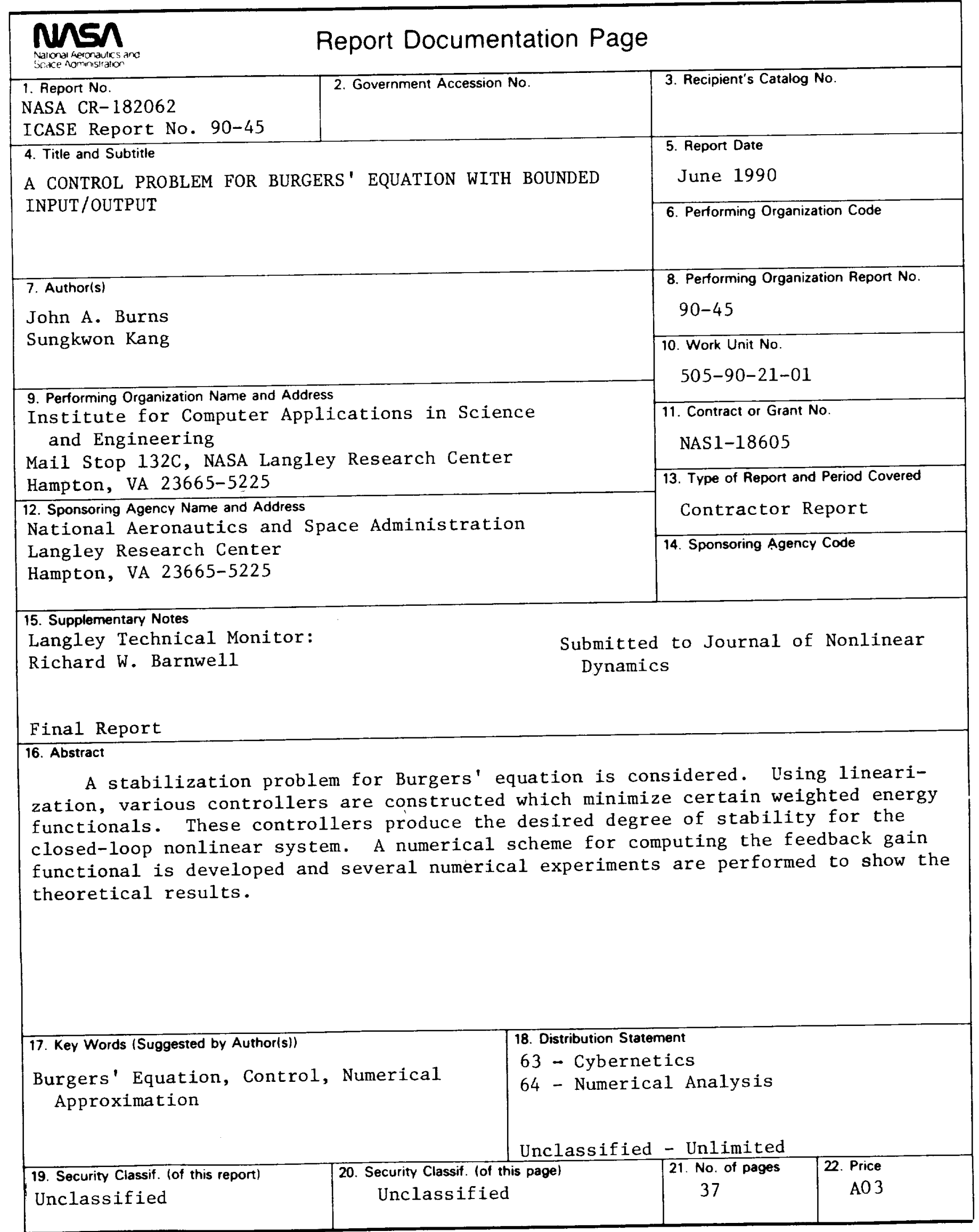


